\title{
A community-driven resource for genomic epidemiology and antimicrobial resistance prediction of Neisseria gonorrhoeae at Pathogenwatch
}

Leonor Sánchez-Busó ${ }^{1,2^{*}}$ (D), Corin A. Yeats ${ }^{1}$, Benjamin Taylor ${ }^{1,3}$, Richard J. Goater ${ }^{3,4}$, Anthony Underwood ${ }^{3}$, Khalil Abudahab ${ }^{3}$, Silvia Argimón ${ }^{3}$, Kevin C. Ma ${ }^{5}$, Tatum D. Mortimer ${ }^{5}$, Daniel Golparian ${ }^{6}$, Michelle J. Cole ${ }^{7}$, Yonatan H. Grad ${ }^{5,8}$, Irene Martin ${ }^{9}$, Brian H. Raphael ${ }^{10}$, William M. Shafer ${ }^{11,12}$, Katy Town ${ }^{10}$, Teodora Wi ${ }^{13}$, Simon R. Harris ${ }^{14}$, Magnus Unemo ${ }^{6}$ and David M. Aanensen ${ }^{1,3^{*}}$

\begin{abstract}
Background: Antimicrobial-resistant (AMR) Neisseria gonorrhoeae is an urgent threat to public health, as strains resistant to at least one of the two last-line antibiotics used in empiric therapy of gonorrhoea, ceftriaxone and azithromycin, have spread internationally. Whole genome sequencing (WGS) data can be used to identify new AMR clones and transmission networks and inform the development of point-of-care tests for antimicrobial susceptibility, novel antimicrobials and vaccines. Community-driven tools that provide an easy access to and analysis of genomic and epidemiological data is the way forward for public health surveillance.

Methods: Here we present a public health-focussed scheme for genomic epidemiology of N. gonorrhoeae at Pathogenwatch (https://pathogen.watch/ngonorrhoeae). An international advisory group of experts in epidemiology, public health, genetics and genomics of $\mathrm{N}$. gonorrhoeae was convened to inform on the utility of current and future analytics in the platform. We implement backwards compatibility with MLST, NG-MAST and NGSTAR typing schemes as well as an exhaustive library of genetic AMR determinants linked to a genotypic prediction of resistance to eight antibiotics. A collection of over 12,000 N. gonorrhoeae genome sequences from public archives has been quality-checked, assembled and made public together with available metadata for contextualization.

(Continued on next page)
\end{abstract}

\footnotetext{
*Correspondence: leo.sanchez-buso@cgps.group; sanchez_leobus@gva.es; david.aanensen@cgps.group

Leonor Sánchez-Busó, Michelle J. Cole, Yonatan H. Grad, Irene Martin, Brian H. Raphael, William M. Shafer, Gianfranco Spiteri, Katy Town, Teodora Wi, Magnus Unemo and David M. Aanensen - Members of the N. gonorrhoeae Pathogenwatch Scientific Steering Group at the time of publication.

${ }^{1}$ Centre for Genomic Pathogen Surveillance, Big Data Institute, Nuffield Department of Medicine, University of Oxford, Oxford, Oxfordshire, UK Full list of author information is available at the end of the article
}

(c) The Author(s). 2021 Open Access This article is licensed under a Creative Commons Attribution 4.0 International License, which permits use, sharing, adaptation, distribution and reproduction in any medium or format, as long as you give appropriate credit to the original author(s) and the source, provide a link to the Creative Commons licence, and indicate if changes were made. The images or other third party material in this article are included in the article's Creative Commons licence, unless indicated otherwise in a credit line to the material. If material is not included in the article's Creative Commons licence and your intended use is not permitted by statutory regulation or exceeds the permitted use, you will need to obtain permission directly from the copyright holder. To view a copy of this licence, visit http://creativecommons.org/licenses/by/4.0/ The Creative Commons Public Domain Dedication waiver (http://creativecommons.org/publicdomain/zero/1.0/) applies to the data made available in this article, unless otherwise stated in a credit line to the data. 


\begin{abstract}
(Continued from previous page)
Results: AMR prediction from genome data revealed specificity values over 99\% for azithromycin, ciprofloxacin and ceftriaxone and sensitivity values around $99 \%$ for benzylpenicillin and tetracycline. A case study using the Pathogenwatch collection of N. gonorrhoeae public genomes showed the global expansion of an azithromycinresistant lineage carrying a mosaic $m$ tr over at least the last 10 years, emphasising the power of Pathogenwatch to explore and evaluate genomic epidemiology questions of public health concern.
\end{abstract}

Conclusions: The N. gonorrhoeae scheme in Pathogenwatch provides customised bioinformatic pipelines guided by expert opinion that can be adapted to public health agencies and departments with little expertise in bioinformatics and lower-resourced settings with internet connection but limited computational infrastructure. The advisory group will assess and identify ongoing public health needs in the field of gonorrhoea, particularly regarding gonococcal AMR, in order to further enhance utility with modified or new analytic methods.

Keywords: Neisseria gonorrhoeae, Pathogenwatch, Public health, Genomics, Epidemiology, Surveillance, Antimicrobial resistance

\section{Background}

Antimicrobial resistance (AMR) is an urgent threat to public health. Neisseria gonorrhoeae, the strictly human pathogen causing the sexually transmitted infection (STI) gonorrhoea, has developed or acquired resistance to the last-line antibiotics used in empiric therapy to treat the infection, and thus has become one of the major global priorities in order to tackle AMR. In 2017, due to the increase in AMR infections and the absence of an effective vaccine, the World Health Organization (WHO) included $N$. gonorrhoeae as a high priority pathogen in need of research and development of new antimicrobials and ideally a vaccine [1]. In 2019, the Centers for Disease Control and Prevention (CDC) again included the gonococcus on the list of urgent threats in the USA [2]. The most recent WHO estimates from 2016 indicate an annual global incidence of 87 million cases of gonorrhoea among adults [3, 4]. Untreated cases can develop complications including an increased acquisition and transmission of HIV. In women, longterm infections can cause infertility, pelvic inflammatory disease, ectopic pregnancy, miscarriage or premature labour [5]. Infections during pregnancy can transmit to newborns at birth causing eye damage that can have permanent effects on vision [6].

Strains of $N$. gonorrhoeae resistant to every recommended treatment have rapidly emerged, including resistance to penicillins, tetracyclines, fluoroquinolones, macrolides and the extended-spectrum cephalosporins (ESCs) [5-8]. The current recommended treatment in many countries is a dual therapy with injectable ceftriaxone plus oral azithromycin, although reports of decreased susceptibility to ceftriaxone as well as azithromycin resistance have increased globally $[7,8]$. One case of failure of dual treatment was reported in 2016 in the United Kingdom (UK) [9]. Additionally, in 2018, a gonococcal strain with resistance to ceftriaxone combined with high-level resistance to azithromycin was detected in both the UK and Australia [10]. The transmission of a ceftriaxoneresistant clone (FC428) has been documented internationally since 2015, raising concerns about the long-term effectiveness of the current treatment in the absence of an available alternative [11]. In some countries such as in Japan, China and since 2019 in the UK, a single dose of ceftriaxone $1 \mathrm{~g}$ is the recommended treatment due to the increasing incidence of azithromycin resistance in $N$. gonorrhoeae and other STI pathogens such as Mycoplasma genitalium [12]. Extensive investigations have been ongoing for years to unveil the genetic mechanisms that explain most of the observed susceptibility patterns for the main classes of antimicrobials for $N$. gonorrhoeae. For ciprofloxacin, nearly all resistant strains have the GyrA S91F amino acid alteration [13-15]; however, resistance prediction from genomic data is not as straightforward for other antibiotics. Known resistance mechanisms often involve additive or suppressive effects as well as epistatic interactions that all together explain just part of the observed phenotypic resistance. For example, there is good evidence that many mosaic structures of the penA gene are associated with decreased susceptibility to ESCs [16, 17]; however, mosaics do not explain all cases of ESC resistance, especially for ceftriaxone, and some mosaic penA alleles do not cause decreased susceptibility or resistance to this antibiotic [16-19]. On top of these, variants that overexpress the MtrCDE efflux pump, mutations in porB1b that reduce drug influx and non-mosaic mutations in penicillin-binding proteins also contribute to decreased susceptibility to ESCs [20]. Furthermore, mutations in the $r p o B$ and $r p o D$ genes, encoding subunits of the RNA polymerase, have been recently related to resistance to ESCs in clinical $N$. gonorrhoeae isolates [21]. Mutations in the 23S rRNA gene (A2045G and C2597T in N. gonorrhoeae nomenclature, coordinates from the WHO 2016 reference panel [22], A2059G and C2611T in Escherichia coli) are frequently associated with azithromycin resistance, as do variants in $m t r R$ or its promoter that increase the expression of the 
MtrCDE efflux pump [5]. Recently, epistatic interactions between a mosaic $m t r$ promoter region and a mosaic $m \operatorname{tr} D$ gene have also been reported to increase the expression of this pump, contributing to macrolide resistance [23, 24]. Mutations in rplD have also been associated with reduced susceptibility to this antibiotic [25], and contrarily, loss-offunction mutations in $m t r C$ have been linked to increased susceptibility to several antibiotics including azithromycin [26]. Thus, we can relatively confidently predict decreased susceptibility or resistance to an antimicrobial using the current known genetic mechanisms; however, phenotypic testing is still necessary to detect resistant cases caused by unknown or novel mechanisms. These inconsistencies with the genomic data will allow the discovery of new mechanisms, which will keep improving the resistance predictions from WGS.

A myriad of methods have been used to discriminate among strains of $N$. gonorrhoeae, from phenotypic to DNA-based techniques [27], but whole genome sequencing (WGS) can provide the complete genome information of a bacterial strain. The cost of amplifying all loci of the different typing schemes via nucleic acid amplification and traditional Sanger sequencing can be more expensive than the cost of WGS of one bacterial genome in many settings. With WGS, multiple genetic AMR mechanisms as well as virulence and typing regions can be targeted simultaneously with the appropriate bioinformatic tools and pipelines. It also provides a significant improvement in resolution and accuracy over traditional molecular epidemiology and typing methods, allowing a genome-wide comparison of strains that can identify AMR clones, outbreaks, transmission networks, national and international spread, known and novel resistance mechanisms as well as also inform on the development of point-of-care tests for antimicrobial susceptibility, novel antimicrobials and vaccines [28, 29]. However, implementation of WGS for genomic surveillance poses practical challenges, especially for low- and middle-income countries (LMICs), due to the need of a major investment to acquire and maintain the required infrastructure.

WGS produces a very high volume of data that needs to be pre-processed and analysed using bioinformatics. Bioinformatics expertise is not always readily available in laboratory and public health settings, and currently, there are no international standards and proficiency trials for which algorithms to use to process WGS data. There are several open-source tools specialised in each step of the pipeline as well as proprietary software containing workflows that simplify the analyses. However, these are less customizable and may not be affordable for all [30, 31]. Choosing the best algorithms and parameters when analysing genomic data is not straightforward as it requires a fair knowledge of the pathogen under study and its genome diversity. Multiple databases containing genetic determinants of AMR for bacterial pathogens are available [30, 31]; however, choosing which one is most complete for a particular organism frequently requires an extensive literature search. Public access web-based species-specific tools and AMR databases revised and curated by experts would be the most approachable option for both well-resourced and LMICs with a reliable internet connection. Very importantly though, the full benefits of using WGS for both molecular epidemiology and AMR prediction can only be achieved if the WGS data are linked to phenotypic data for the gonococcal isolates and, as much as feasible, clinical and epidemiological data for the patients.

Here, we present a public health-focussed system to facilitate genomic epidemiology of N. gonorrhoeae within Pathogenwatch (https://pathogen.watch/ngonorrhoeae), which includes the latest analytics for typing, detection of genetic AMR determinants and prediction of AMR from $N$. gonorrhoeae genome data, linked to metadata where available, as well as a collection of over 12,000 gonococcal genomes from public archives for contextualization. We formed an advisory group including experts in the field of $N$. gonorrhoeae epidemiology, public health, AMR, genetics and genomics to consult on the development and design of the tool, such as the analytics and genetic AMR mechanisms to include, in order to adapt the platform for ongoing public health needs. We present this scheme as a community-steered model for genomic surveillance that can be applied to other pathogens.

\section{Methods}

\section{The Pathogenwatch platform: technical summary}

Pathogenwatch is a web-based platform with several different components. The main interface is a React [32] single-page application with a style based on Material Design Lite [33]. Phylogenetic trees are plotted using Phylocanvas [34], maps using Leaflet [35] and networks with Sigma [36]. The back end is written in Node.js and contains an API service for the user interface and four 'Runner' services for the following analyses: species prediction, single-genome analyses, tree building and core genome multi-locus sequence typing (cgMLST) clustering. Docker containers are used for queuing tasks, streaming input or result files through standard input and storing JSON data from standard output. A MongoDB cluster is used for data storage and task queuing/ synchronisation. Pathogenwatch shares some visualisation components with Microreact [37], such as those associated with the phylogenetic tree and the map. However, Pathogenwatch includes an analytical framework which is unique to this platform. 
Generation of the $\boldsymbol{N}$. gonorrhoeae core genome library Pathogenwatch implements a library of core genome sequences for several supported organisms. In the case of $N$. gonorrhoeae, a core gene set was built from the 14 complete reference genomes that constitute the 2016 WHO reference strain panel [22] using the pangenome analysis tool Roary [38] as described in Harris et al. [15]. Briefly, the minimum percentage of identity for blastp was set to $97 \%$, and the resulting core genes were aligned individually using MAFFT. The resulting genes with a percentage of identity above $99 \%$ were postprocessed as described in [39]. Representatives for each family were selected by choosing the sequence with the fewest differences to the others on average and searched using tblastn (percentage of identity $\geq 80 \%, E$-value $\leq 1 \mathrm{e}$ $-35)$ against the 14 high-quality reference genomes. Families without a complete match in every reference (100\% coverage) or that had multiple matches were removed. Overlapping genes from each reference were merged into pseudocontigs and grouped by gene composition. For each family, a representative was selected as before and searched/filtered using the references as before. The final core gene set contains 1542 sequences that span a total of 1,470,119 nucleotides (approximately $67 \%$ of a typical $N$. gonorrhoeae genome length, $2.2 \mathrm{Mb}$ ). A BLAST database was constructed from these core segments and used to profile new assemblies.

\section{Profiling new assemblies}

New genome assemblies can be uploaded by a user (drag and drop) or calculated from high-throughput shortread data directly within Pathogenwatch using SPAdes [40] as described in [41].

A taxonomy assignment step for species identification is performed on the uploaded assemblies by using Speciator [42]. New assemblies are then queried against a species-specific BLAST database using blastn. For $N$. gonorrhoeae, every core locus needs to match at least $80 \%$ of its length to be considered as present. Further filtering steps are applied to remove loci that can be problematic for tree building, such as paralogs or loci with unusually large number of variant sites compared to an estimated substitution rate on the rest of the genome, as described in [43]. The overall substitution rate is calculated as the number of total differences in the core library divided by the total number of nucleotides. Indels are ignored to minimise the noise that could be caused by assembly or sequencing errors. The expected number of substitutions per locus is determined by multiplying this substitution rate by the length of the representative sequence.

The number of substitutions observed for each locus between the new assembly and the reference sequence are scaled to the total number of nucleotides that match the core library, creating a pairwise score that is saved on a distance matrix and is used for Neighbour-Joining tree construction, as described in [44].

\section{Algorithms for sequence typing and cgMLST clustering}

Alleles and sequence types (STs) for multi-locus sequence typing (MLST) [45] and cgMLST ( $N$. gonorrhoeae cgMLST v1.0) [46] were obtained from PubMLST $[47,48]$, for $N$. gonorrhoeae multi-antigen sequence typing (NG-MAST) [49] from [50] and for $N$. gonorrhoeae sequence typing for antimicrobial resistance (NG-STAR) [51] from [52] (Table 1). A search tool implemented as part of Pathogenwatch is used to make the assignments for MLST, cgMLST and NG-STAR, while NGMASTER [54] is used for NGMAST. Briefly, exact matches to known alleles are searched for, while novel sequences are assigned a unique identifier. The combination of alleles is used to assign a ST as described in [55]. Databases are regularly updated and novel alleles and STs should be submitted by the user to the corresponding schemes for designation.

cgMLST typing information is used for clustering individual genomes with others in the Pathogenwatch database using single linkage clustering as described in [56]. Users can select the clustering threshold (i.e. number of loci with differing alleles), and a network graph based on the SLINK [57] algorithm is calculated within individual genome reports.

Table $1 \mathrm{~N}$. gonorrhoeae sequence typing schemes implemented in Pathogenwatch

\begin{tabular}{|c|c|c|c|c|}
\hline Typing scheme $^{a}$ & Loci (number) & Note & Pathogenwatch implementation & References \\
\hline cgMLST & $(N=1649)$ & N. gonorrhoeae cgMLST v1.0 & $\begin{array}{l}\text { Typing algorithm, database from } \\
\text { PubMLST }\end{array}$ & {$[46-48,53]$} \\
\hline MLST & $\begin{array}{l}\text { abcZ, adk, aroE, fumC, gdh, } \\
\text { pdhC, pgm }(N=7)\end{array}$ & Housekeeping genes in Neisseria spp. & $\begin{array}{l}\text { In-house typing tool, database from } \\
\text { PubMLST }\end{array}$ & {$[45,47,48,53]$} \\
\hline NG-MAST & porB, tbpB $(N=2)$ & $\begin{array}{l}\text { Genes encoding highly-variable } \\
\text { membrane proteins }\end{array}$ & $\begin{array}{l}\text { NG-MASTER, database from NG-MAST } \\
\text { website }\end{array}$ & {$[49,50,54]$} \\
\hline NG-STAR & $\begin{array}{l}\text { penA, } m t r R, \text { porB, ponA, } \\
\text { gyrA, parC, } 235 \text { rDNA }(N=7)\end{array}$ & $\begin{array}{l}\text { Genes involved in antimicrobial } \\
\text { resistance }\end{array}$ & $\begin{array}{l}\text { In-house typing tool, database from } \\
\text { NG-STAR website }\end{array}$ & {$[51-53]$} \\
\hline
\end{tabular}

a Typing scheme: cgMLST core genome multi-locus sequence typing, MLST multi-locus sequence typing, NG-MAST N. gonorrhoeae multi-antigen sequence typing, NG-STAR N. gonorrhoeae sequence typing for antimicrobial resistance 


\section{AMR library and detection of genetic AMR determinants} Genes and point mutations (single-nucleotide polymorphisms (SNPs) and indels) were detected using Pathogenwatch AMR v2.4.9 [58]. Pathogenwatch AMR also provides a prediction of AMR phenotype inferred from the combination of identified mechanisms. Genetic determinants described in the literature as involved in AMR in $N$. gonorrhoeae were collated into a library in TOML format (version 0.0.11). A test dataset containing 3987 isolates from 13 studies [15, 18, 22, 59-68] (Additional file 1: Table S1) providing minimum inhibitory concentration (MIC) information for six antibiotics (benzylpenicillin, tetracycline, ciprofloxacin, cefixime, ceftriaxone and azithromycin) was used to benchmark and to curate this library. A validation benchmark was posteriorly run with a dataset of 1607 isolates from 3 other publications [69-71] with MIC information for the same six antibiotics plus spectinomycin (Additional file 1: Table S1). EUCAST clinical breakpoints v9.0 [72] were used to define susceptibility (S), susceptibility with an increased exposure (I) or resistance (R) (SIR) categorical interpretations of MICs for all antibiotics except for azithromycin, for which the EUCAST epidemiological cut-off (ECOFF) was used to define non-susceptibility/resistance (ECOFF $>1 \mathrm{mg} / \mathrm{L})$. As a result of the benchmark analyses, sensitivity, specificity and positive/negative predictive values (PPV/NPV) were obtained for the AMR mechanisms implemented in the library and, globally, for each of the antibiotics. Confidence intervals (95\%) for these statistics were calculated using the epi.tests function in the epiR R package v1.0-14 [73]. Individual or combined AMR mechanisms with a PPV below 15\% were discarded from the library to optimise the overall predictive values. Visual representations of the observed ranges of MIC values for a particular antibiotic for each of the observed combinations of genetic AMR mechanisms on the test dataset were used to identify and assess combinations of mechanisms that have an additive or suppressive effect on AMR. These were included in the library.

As part of the accuracy testing of the AMR library, we ran the 2016 WHO $N$. gonorrhoeae reference genomes 2016 panel $(n=14)$ through Pathogenwatch and compared the detected list of genetic AMR mechanisms with the list published in the original study [22]. For the WHO U strain, a discrepancy on a mutation in parC was further investigated by mapping the original raw Illumina data (European Nucleotide Archive (ENA) run accession ERR449479) to the reference genome assembly (ENA genome accession LT592159.1) and visualised using Artemis [74].

In short-read assemblies, the four copies of the $23 \mathrm{~S}$ rRNA gene are collapsed into one, thus the detection of the A2045G and C2597T mutations is dependent on the consensus bases resulting from the number of mutated copies $[64,67,75]$.
Quality check and assembly of public sequencing data Public N. gonorrhoeae genomes with geolocation data were obtained from the ENA in November 2019. This list was complemented by an exhaustive literature search of studies on $N$. gonorrhoeae genomics without metadata submitted to the ENA but instead made available as supplementary information in the corresponding publications. Raw paired-end short-read data from a list of 12, 192 isolates was processed with the GHRU assembly pipeline v1.5.4 [76]. This pipeline runs a Nextflow workflow to quality-check (QC) paired-end short-read fastq files before and after filtering and trimming, assembles the data and quality-checks the resulting assembly. Results from the pipeline are provided in Additional file 2. In this pipeline, QC of short reads was performed using FastQC v0.11.8 [77]. Trimming was done with Trimmomatic v0.38 [78] by cutting bases from the start and end of reads if they were below a Phred score of 25, trimming using a sliding window of size 4 and cutting once the average quality within the window fell below a Phred score of 20. Only reads with length above a third of the original minimum read length were kept for further analyses. After trimming, reads were corrected using the kmer-based approach implemented in Lighter v1.1.1 [79] with a kmer length of $32 \mathrm{bp}$ and a maximum number of corrections allowed within a 20-bp window of 1 . ConFindr v0.7.2 was used to assess intra- and inter-species contamination [80]. Mash v2.1 [81] was applied to estimate genome size using a kmer size of $32 \mathrm{bp}$ and Seqtk v1.3 [82] to down sample fastq files if the depth of coverage was above $100 \times$. Flash v1.2.11 [83] was used to merge reads with a minimum overlap length of $20 \mathrm{bp}$ and a maximum overlap of $100 \mathrm{bp}$ to facilitate the subsequent assembly process. SPAdes v3.12 [40] was used for genome assembly with the --careful option selected to reduce the number of mismatches and short indels with a range of kmer lengths depending on the minimum read length. The final assemblies were quality-checked using Quast v5.0.2 [84] and ran through the species identification tool Bactinspector [85]. QC conditions were assessed and summarised using Qualifyr [86].

Fastq files with poor quality in which the trimming and filtering step discarded all reads from either one, or both pairs were excluded from the analyses because the assembly pipeline is optimised for paired-end data. Assemblies with an N50 below 25,000 bp, a number of contigs above 300 , a total assembly length above $2.5 \mathrm{Mb}$ or a percentage of contamination above $5 \%$ were also excluded.

\section{Metadata for public genomes}

Geolocation data (mainly country), collection dates (day, month and year when available), ENA project accession and associated PubMed ID were obtained from the ENA API for all the genomes in the pipeline [87]. A manual 
extensive literature search was performed to identify the publications containing the selected genomes. In order to complete published studies as much as possible, additional genomes were downloaded and added to the dataset. Metadata for the final set was completed with the information contained in supplementary tables on the corresponding publications, including phenotypic antimicrobial susceptibility data. Submission date was considered instead of collection date when the latter was not available; however, this occurred in only a few cases $(<0.5 \%)$.

\section{Creation of the $N$. gonorrhoeae Pathogenwatch Scientific Steering Group}

International experts in the field of $N$. gonorrhoeae AMR, microbiology, genetics, genomics, epidemiology and public health were approached and agreed to participate as members of the ' $N$. gonorrhoeae Pathogenwatch Scientific Steering Group' in order to discuss the analytics in Pathogenwatch and make sure they met the current needs of the public health and scientific community. During the updates made to the platform and the preparation of this manuscript, these experts participated in virtual sessions to discuss the list of genetic AMR determinants and their association with SIR categories (Table 2) based on experimental and/or computational evidence. Some of the members of the group had previously been directly involved in many of these studies. Other current and future updates were also discussed, such as the inclusion of the NG-STAR typing scheme [51] and the organisation of published genomes into public collections, data sharing, privacy and the interconnectivity of Pathogenwatch with other platforms, such as PubMLST [48] or the ENA. The group will regularly discuss new updates to the platform.

\section{Data sharing and privacy}

Sequencing data and metadata files uploaded to Pathogenwatch by the user are kept within the user's private account. Genomes can be grouped into collections and these can be toggled between private and accessible to collaborators via a URL. Collection URLs include a 12-letter random string to secure them against brute force searching. Setting a collection to 'off-line mode' allows users to work in challenging network conditions, which may be beneficial in LMICs-all data are held within the browser. Users can also integrate private and potentially confidential metadata into the display without uploading it to the Pathogenwatch servers (locally within the browser on a user's machine).

\section{Results}

N. gonorrhoeae genome analytics in Pathogenwatch Pathogenwatch is a web-based platform for epidemiological surveillance using genome sequencing data. After upload, different analytics are run simultaneously (Fig. 1): cgMLST [46], MLST [45], NG-MAST [49] and NGSTAR [51] typing schemes (Table 1), a genotypic prediction of phenotypic resistance using a customised AMR library (Table 2) that includes known genetic AMR mechanisms for 8 antimicrobials, as well as statistics on the quality of the assemblies (Additional file 3: Fig. S1). These analytical features differentiate Pathogenwatch from a parallel platform from the same group, Microreact [37], which shares one of the main layouts with Pathogenwatch (a phylogenetic tree, a map and a table or timeline), but it is intended for visualisation of precomputed phylogenetic trees with accompanying metadata, while Pathogenwatch also includes analytical tools.

Genomes from one or multiple studies can be grouped into collections (Fig. 2 and Additional file 3: Fig. S2), and the genomic data are automatically processed by comparing with a core $N$. gonorrhoeae genome built from WHO reference strain genomes [15, 22]. A phylogenetic tree is obtained as a result, representing the genetic relationship among the isolates in the collection. Metadata can be uploaded at the same time as the genome data, and if location coordinates for the isolates are provided, this information is plotted into a map (Fig. 2 and Additional file 3: Figs. S1 and S2). If date or year of isolation is also provided, this information is represented in a timeline. The three panels on the main collection layout-the tree, the map and a table or timeline-are functionally integrated so filters and selections made by the user update all of them simultaneously. Users can also easily switch among the metadata and the results of the main analytics: typing, genome assembly statistics, genotypic AMR prediction, AMR-associated SNPs, AMR-associated genes and the timeline (Additional file 3: Fig. S1). cgMLST is used for finding close genomes in the Pathogenwatch database based on allele differences to one individual isolate (Additional file 3: Fig. S3). A video demonstrating the usage and main features of Pathogenwatch is available [113]. Notes on data sharing and privacy are available in the 'Methods' section.

\section{Library of genetic AMR mechanisms: genotypic and phenotypic benchmarks}

We compiled genetic AMR mechanisms previously reported for $N$. gonorrhoeae up to the writing of this manuscript into the AMR library in Pathogenwatch (Table 2). A genotypic accuracy testing of the AMR library was performed using the $14 \mathrm{~N}$. gonorrhoeae reference genomes from the WHO 2016 panel [22], which were uploaded into Pathogenwatch. All the genetic AMR determinants described as present in these isolates in the original publication and implemented in the Pathogenwatch AMR library were obtained as a result (Additional file 1: Table S2). Only one discrepancy was 
Table 2 List of $N$. gonorrhoeae genetic antimicrobial resistance (AMR) determinants in Pathogenwatch. References that report evidence of association of each mechanism with AMR in clinical isolates and/or where their role on AMR has been confirmed in the laboratory through, e.g. transformation experiments, are included in the table. Effect: $R=$ resistance, I= susceptibility but increased exposure, $A$ = additive effect, $N$ = negative effect. $R$ and I follow the EUCAST clinical breakpoints except for azithromycin, for which the epidemiological cut-off (ECOFF) is reported and used instead

\begin{tabular}{|c|c|c|c|}
\hline Antibiotic (MIC breakpoint mg/L) & Genetic AMR determinants & Effect & Evidence (references) \\
\hline \multirow{18}{*}{$\begin{array}{l}\text { Azithromycin } \\
\text { (R: MIC>1, ECOFF) }\end{array}$} & $23 \mathrm{~S}$ rDNA $2045 \mathrm{~A}>\mathrm{G}$ substitution (2059A>G in E. coli) & $\mathrm{R}$ & {$[75]$} \\
\hline & $23 \mathrm{~S}$ rDNA $2597 \mathrm{C}>\mathrm{T}$ substitution $(2611 \mathrm{C}>\mathrm{T}$ in E. coli) & $\mathrm{R}$ & {$[88]$} \\
\hline & ermA, ermB, ermC, ermF genes & $\mathrm{R}$ & {$[89,90]$} \\
\hline & ereA, ere $B$ genes & $\mathrm{R}$ & {$[22]$} \\
\hline & mefA gene & $\mathrm{R}$ & {$[90,91]$} \\
\hline & mac $A B$ promoter $-48 \mathrm{G}>T$ substitution ${ }^{a}$ & $\mathrm{R}$ & {$[92]$} \\
\hline & mtrR promoter mosaic ${ }^{\mathrm{b}}$ & & \\
\hline & N. meningitidis-like mosaic $(n=1)$ & $\mathrm{R}$ & {$[23]$} \\
\hline & N. lactamica-like mosaic $(n=2)$ & $\mathrm{R}$ & {$[23]$} \\
\hline & $m t r D$ mosaic ${ }^{b}$ & & \\
\hline & N. meningitidis-like mosaic $(n=1)$ & $\mathrm{R}$ & {$[23]$} \\
\hline & N. lactamica-like mosaic $(n=2)$ & $\mathrm{R}$ & {$[23]$} \\
\hline & $m t r R$ promoter -57 delA $A^{a}$ & A & {$[93,94]$} \\
\hline & $m t r R$ G45D & A & {$[95,96]$} \\
\hline & $m t r C$ loss-of-function & N & {$[26]$} \\
\hline & rp/V ARAK tandem duplication (position 90) & $\mathrm{R}$ & {$[18]$} \\
\hline & rp/V KGPSLK tandem duplication (position 83) & $\mathrm{R}$ & {$[18]$} \\
\hline & rp/D G70D & A & {$[25]$} \\
\hline \multirow{4}{*}{$\begin{array}{l}\text { Ceftriaxone } \\
\text { (R: } M I C>0.125)\end{array}$} & penA mosaic (A311V, I312M, V316P/T, T483S and G545S) & $\mathrm{R}$ & {$[97,98,100]$} \\
\hline & penA V316P, T483S, A501PN, G542S & $\mathrm{R}$ & {$[97,100]$} \\
\hline & rpoB P157L, G158V, R201H & $\mathrm{R}$ & {$[21]$} \\
\hline & rpoD D92-95 deletion, E98K & । & {$[21]$} \\
\hline \multirow{6}{*}{$\begin{array}{l}\text { Cefixime } \\
\text { (R: MIC>0.125) }\end{array}$} & $m t r R$ G45D & A & {$[95,96]$} \\
\hline & penA mosaic (I312M, V316T, G545S) & $\mathrm{R}$ & {$[97,98,100]$} \\
\hline & penA mosaic (A311V, I312M, V316P/T, T483S and G545S) & $\mathrm{R}$ & {$[97,98,100]$} \\
\hline & penA V316P, T483S, A501P & । & {$[97,100]$} \\
\hline & rpoB P157L, G158V, R201H & । & {$[21]$} \\
\hline & rpoD D92-95 deletion, E98K & I & {$[21]$} \\
\hline \multirow{6}{*}{$\begin{array}{l}\text { Ciprofloxacin } \\
\text { (I: } 0.03<\text { MIC } \leq 0.06 \text {; } \\
\text { R: MIC }>0.06)\end{array}$} & gyrA S91F, D95A/N & R & [99] \\
\hline & gyrA D95G & । & [99] \\
\hline & norM promoter $-7 A>G,-104 C>T$ substitutions ${ }^{a}$ & । & {$[101]$} \\
\hline & parC D86N, S87R & R & [99] \\
\hline & $\operatorname{parC~S871/N,~S88P,~E91K~}$ & । & [99] \\
\hline & parE G410V & । & [102] \\
\hline \multirow{5}{*}{$\begin{array}{l}\text { Tetracycline } \\
(\mathrm{I}: 0.5<\mathrm{MIC} \leq 1 \text {; } \\
\text { R: } \mathrm{MIC}>1)\end{array}$} & $m \operatorname{trR}$ A39T, G45D & A & {$[95,96]$} \\
\hline & mtrR loss-of-function & । & {$[22]$} \\
\hline & $m t r R$ promoter $-56 \mathrm{~A}>C$ substitution, -57 delA deletion ${ }^{a}$ & । & {$[23,93,94]$} \\
\hline & mtrR promoter $-131 \mathrm{G}>\mathrm{A}(\mathrm{mtrC}-120 \mathrm{G}>\mathrm{A} \text { substitution, } m \operatorname{tr} 120)^{\mathrm{a}}$ & । & [95] \\
\hline & rps J V57M & । & [103] \\
\hline
\end{tabular}


Table 2 List of N. gonorrhoeae genetic antimicrobial resistance (AMR) determinants in Pathogenwatch. References that report evidence of association of each mechanism with AMR in clinical isolates and/or where their role on AMR has been confirmed in the laboratory through, e.g. transformation experiments, are included in the table. Effect: $R=$ resistance, $I=$ susceptibility but increased exposure, $\mathrm{A}$ = additive effect, $N$ = negative effect. $\mathrm{R}$ and I follow the EUCAST clinical breakpoints except for azithromycin, for which the epidemiological cut-off (ECOFF) is reported and used instead (Continued)

\begin{tabular}{|c|c|c|c|}
\hline Antibiotic (MIC breakpoint $\mathrm{mg} / \mathrm{L}$ ) & Genetic AMR determinants & Effect & Evidence (references) \\
\hline & tetM gene & $\mathrm{R}$ & [104] \\
\hline \multirow{10}{*}{$\begin{array}{l}\text { Penicillins } \\
(\mathrm{I}: 0.06<\mathrm{MIC} \leq 1 \text {; } \\
\mathrm{R}: \mathrm{MIC}>1)\end{array}$} & blaTEM gene & $\mathrm{R}$ & {$[105]$} \\
\hline & $m t r R$ G45D & I & {$[95,96]$} \\
\hline & mtrR A39T & A & {$[95]$} \\
\hline & $m t r R$ loss-of-function & । & {$[22]$} \\
\hline & $m$ trR promoter $-56 \mathrm{~A}>\mathrm{C},-57$ del $\mathrm{A}^{\mathrm{a}}$ & । & {$[23,94]$} \\
\hline & mtrR promoter $-131 \mathrm{G}>\mathrm{A}(\mathrm{mtrC}-120 \mathrm{G}>\mathrm{A} \text { substitution, } m \operatorname{tr} 120)^{\mathrm{a}}$ & । & [95] \\
\hline & penA I312M, V316P/T, ins346D, T483S, A501P/TN, G542S, G545S, P551S & 1 & {$[97,100]$} \\
\hline & penA mosaic (I312M, V316T, G545S) & A & {$[97,98,100]$} \\
\hline & ponA1 L421P & I & [106] \\
\hline & porB1b G120K, A121N/D & । & [107] \\
\hline \multirow{3}{*}{$\begin{array}{l}\text { Spectinomycin } \\
\text { (R: MIC>64) }\end{array}$} & 16S rDNA 1184C>T (1192C>T in E. coli) & $\mathrm{R}$ & [108] \\
\hline & rpsE T24P & $\mathrm{R}$ & [109] \\
\hline & rpsE V27- deletion, K28E & R/A & [109] \\
\hline Sulfonamides ${ }^{e}$ & folP R228S & $\mathrm{R}$ & {$[22,110]$} \\
\hline
\end{tabular}

${ }^{a}$ Nomenclature of the mutations on the $\operatorname{mac} A B, m t r R$ and norM promoter regions is based on $N$. gonorrhoeae coordinates considering the distance from the start of the $\operatorname{mac} A B, \operatorname{mtr} R$ and norM genes, respectively. ${ }^{\mathrm{b}}$ Note that mosaics are caused by recombination events, which can have variable breakpoints with different effects on azithromycin MIC if any. In this version, we have included the three mosaics described by Wadsworth et al. [23], but the list will be expanded as new mosaic $m t r$ (intergenic region between $m t r R$ and $m t r C$ ) and $m t r D$ alleles having an effect on azithromycin MICs are published. ${ }^{\circ}$ The list of genetic AMR mechanisms for the ESCs ceftriaxone and cefixime does not include all known porB1b or mtrR-associated variants as their effect was found not to be relevant in increasing MIC on the benchmark analyses for phenotypic AMR prediction purposes despite the experimental evidence reported in Zhao et al. [111]. In the case of strains carrying penA-associated mutations, their immediate predicted phenotype is that of those carrying penA-associated variants. ${ }^{\mathrm{d}}$ The list of genetic AMR mechanisms for tetracycline does not include porB1b mutations as their effect was found not to be relevant in increasing MIC on the benchmark analyses for

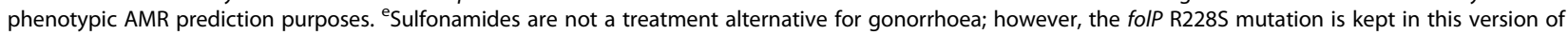
the library for surveillance purposes

found. The WHO U strain was reported as carrying a parC S87W mutation. However, mapping the original Illumina data from this isolate with the final genome assembly revealed that this strain carries a wild type allele (Additional file 3: Fig. S4). MLST and NG-MAST types were the same as those reported in the original publication (note that NG-STAR was not available at that time) and the porA mutant gene was found in WHO $\mathrm{U}$ as previously described. This mutant porA has nearly a 95\% nucleotide identity to Neisseria meningitidis and $89 \%$ to N. gonorrhoeae, and it is included as screening because it has previously been shown to cause false negative results in some molecular detection tests for N. gonorrhoeae [114].

Then, we also performed a genotypic-phenotypic benchmark using a test dataset of $3987 N$. gonorrhoeae isolates from 13 different studies containing MIC information for at least part of the following six antibiotics: ceftriaxone, cefixime, azithromycin, ciprofloxacin, benzylpenicillin and tetracycline (Additional file 1: Table S1). EUCAST clinical breakpoints were applied for five of the antimicrobials except for azithromycin, for which the adoption of an ECOFF $>1 \mathrm{mg} / \mathrm{L}$ is now recommended to distinguish isolates with azithromycin resistance determinants, instead of a clinical resistance breakpoint [115, 116]. A visualisation of the range of MICs on each particular combination of genetic AMR mechanisms observed on the isolates from the benchmark test dataset (Fig. 3a, b and Additional file 3: Figs. S5-S10) revealed combinations that show an additive effect on AMR. These combinations were included in the AMR library to improve the accuracy of the genotypic prediction. For example, rpsJ V57M and some $m t r R$-associated mutations individually are associated with a decreased susceptibility or intermediate resistance to tetracycline (MICs of $0.5-1 \mathrm{mg} / \mathrm{L}$ ); however, a combination of these variants can increase MICs above the EUCAST resistance breakpoint for tetracycline (MICs $>1 \mathrm{mg} / \mathrm{L}$ ) (Additional file 3: Fig. S9). This is the case of the combination of rpsJ V57M with the mtrR promoter -57delA mutation ( $N=681$ isolates, $94.9 \%$ positive predictive value, PPV) or with $m t r R$ promoter -57 delA and $m t r R$ G45D ( $N=83$ isolates, 93.9\% PPV). Several combinations of penA, ponA1, $m$ trR and porB1b mutations were observed to be able to increase the benzylpenicillin MIC 


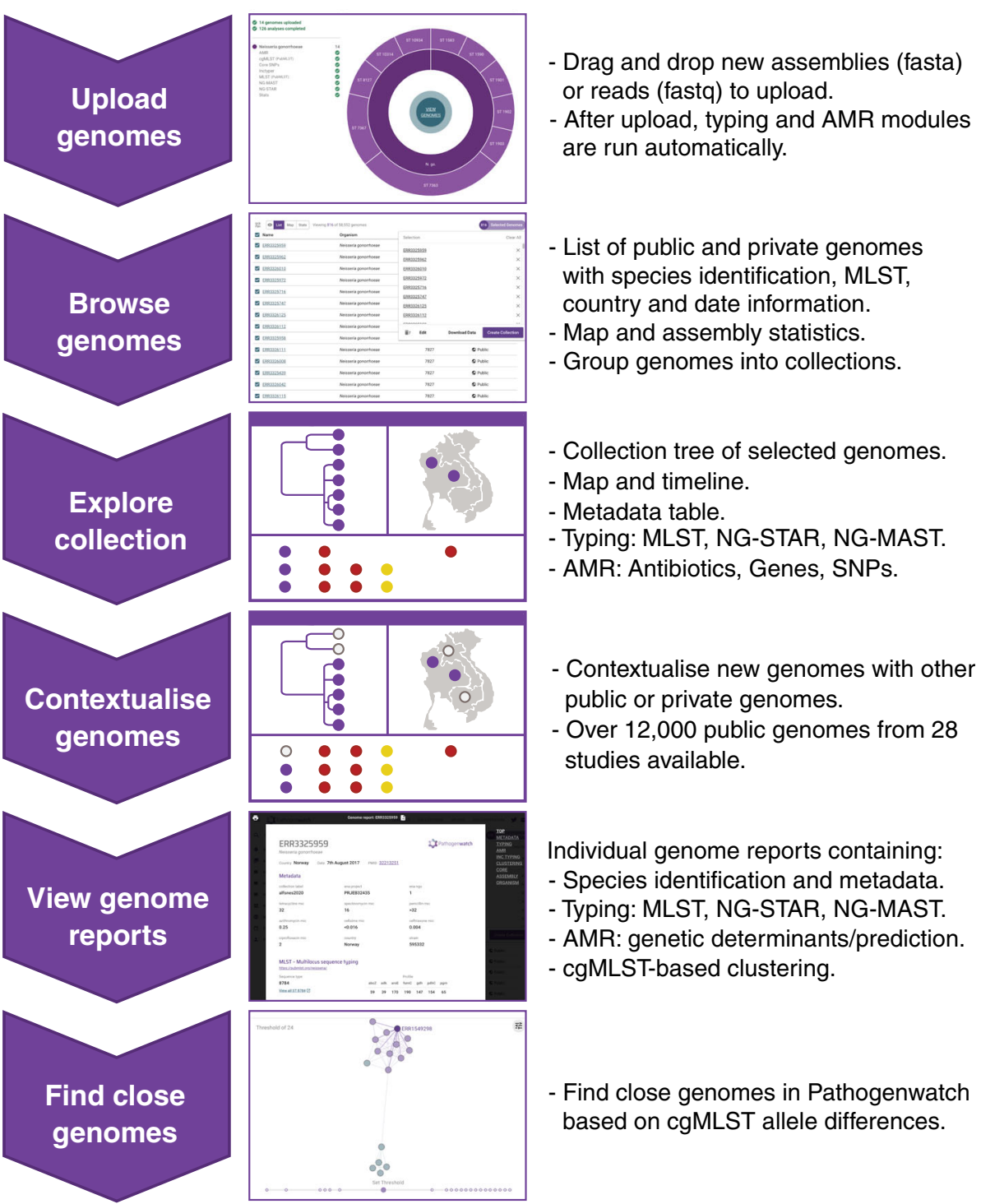

Fig. 1 Main workflow in Pathogenwatch. New genomes can be uploaded and combined with public data for contextualisation. The collection view allows data exploration through a combined phylogenetic tree, a map, a timeline and the metadata table, which can be switched to show typing information (multi-locus sequence typing, MLST; N. gonorrhoeae sequence typing for antimicrobial resistance, NG-STAR; and N. gonorrhoeae multi-antigen sequence typing, NG-MAST) as well as known genetic AMR mechanisms for eight antibiotics. Genome reports summarise the metadata, typing and AMR marker results for individual isolates and allow finding other close genomes in Pathogenwatch based on core genome MLST (cgMLST). SNPs: single-nucleotide polymorphisms

above the resistant threshold in most of the cases (Additional file 3: Fig. S10). This is the case of the porB1b mutations combined with $m$ trR A39T $(N=31$ isolates, $100 \%$ PPV), with the mtrR promoter -57delA deletion $(N=286$ isolates, $96.5 \% \mathrm{PPV})$ or with $m t r R$ promoter -57delA and ponA1 L421P ( $N=269$ isolates, 96.3\%). Despite mosaic pen $A$ not being a main driver of resistance to penicillins, a combination of the porB $1 b$ mutations with the three main mosaic penA mutations (G545S, I312M and $\mathrm{V} 316 \mathrm{~T}$ ) was also associated with a resistant phenotype in all cases $(N=17$ isolates, $100 \%$ PPV). A recent publication showed that loss-of-function mutations in $m t r C$ increased susceptibility to azithromycin and are associated with isolates from the cervical environment [26]. We included the presence of a disrupted $m \operatorname{tr} C$ as a modifier of antimicrobial susceptibility in the presence of an $m$ tr mosaic, as we did not have enough evidence from the test dataset to assess the MIC ranges of isolates with the 23S rDNA A2045G and C2597T mutations with and without a disrupted $m t r C$ gene.

Results from the benchmark (Additional file 1: Table S3) show sensitivity values (true positive rates, $\mathrm{TP} /(\mathrm{TP}+$ 


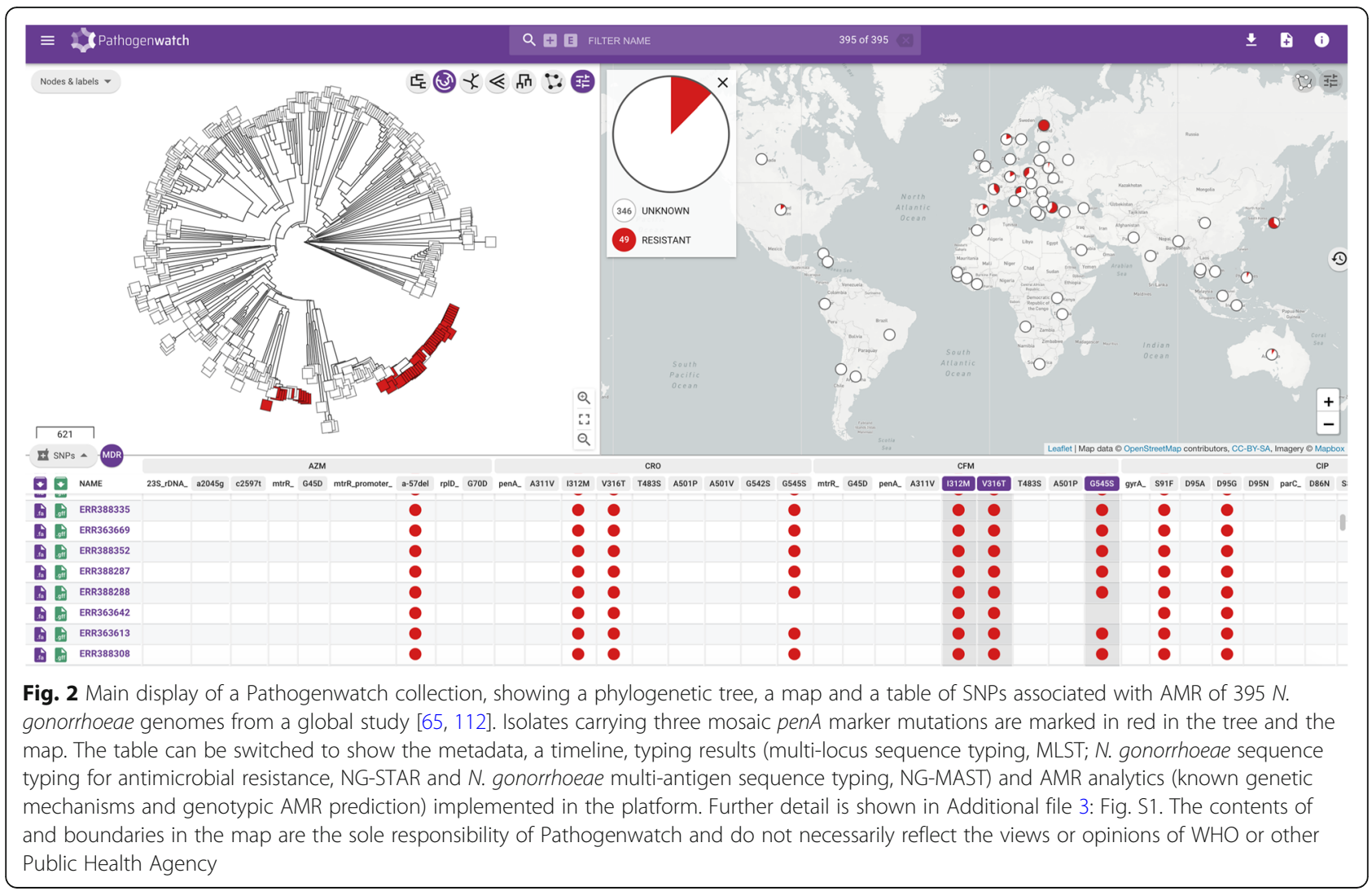

$\mathrm{FN}) ; \mathrm{TP}=$ true positives, $\mathrm{FN}=$ false negatives) above $96 \%$ for tetracycline (99.2\%), benzylpenicillin (98.1\%), ciprofloxacin $(97.1 \%)$ and cefixime $(96.1 \%)$, followed by azithromycin (71.6\%) and ceftriaxone (33.3\%). These results reflect the complexity of the resistance mechanisms for azithromycin and ceftriaxone, where the known genetic determinants explain only part of the antimicrobial susceptibility. However, specificity values (true negative rates, $\mathrm{TN} /(\mathrm{TN}+\mathrm{FP}) ; \mathrm{TN}=$ true negatives, $\mathrm{FP}=$ false positives) for these two antibiotics as well as ciprofloxacin were above 99\% (Additional file 1: Table S3), demonstrating that the genetic mechanisms included in the database have a role in AMR. The specificity value for cefixime was lower but nearly $90 \%$, mainly due to the high number of isolates with an MIC below the threshold but with three mutations characterising a mosaic penA allele (G545S, I312M and V316T, $\mathrm{TP}=367, \mathrm{TN}=323, \mathrm{PPV}=53.2 \%$; Additional file 1: Table S4). Benzylpenicillin and tetracycline showed specificity values of $77.3 \%$ and $61.3 \%$, respectively. In the first case, all the mechanisms included in the library showed a PPV value above 94\%. For tetracycline, a considerable number of false positive results are mainly caused by the presence of rpsJ $\mathrm{V} 57 \mathrm{M}$, for which $\mathrm{PPV}=83.8 \% \quad(\mathrm{TP}=1083, \mathrm{FP}=209$; Additional file 1: Table S4). However, this mutation was kept in the AMR library because it can cause intermediate resistance to tetracycline on its own (Additional file 3: Fig. S9).

Results from the benchmark analysis on the 3987isolate dataset were used to curate and optimise the AMR library. Thus, in order to objectively validate it, the benchmark analysis was also run on a combination of three different collections $(N=1607$, Additional file 1: Table S1) with available MIC information for seven antibiotics including spectinomycin (Additional file 1: Table S3) [70, 71, 117]. Results from the test and validation benchmark runs were compared, showing that sensitivity values on the six overlapping antibiotics were very similar, with the validation benchmark performing even better for azithromycin and ceftriaxone (Fig. 3c). In terms of specificity, both datasets performed equally well for all antibiotics except for benzylpenicillin, in which specificity drops in the validation benchmark. This is due to the penA_ins346D mutation ( TP $=1125$, $\mathrm{FP}=83)$ and the blaTEM genes $(\mathrm{TP}=525, \mathrm{FP}=36)$, which despite showing false positives, have a PPV above 93\% (Additional file 1: Table S5). In general, discrepancies found between the test and the validation benchmarks can be explained by particular mechanisms that on their own show high predictive values and affect antibiotics for which we do not currently understand all the factors involved in resistance, such as azithromycin and the ESCs. 


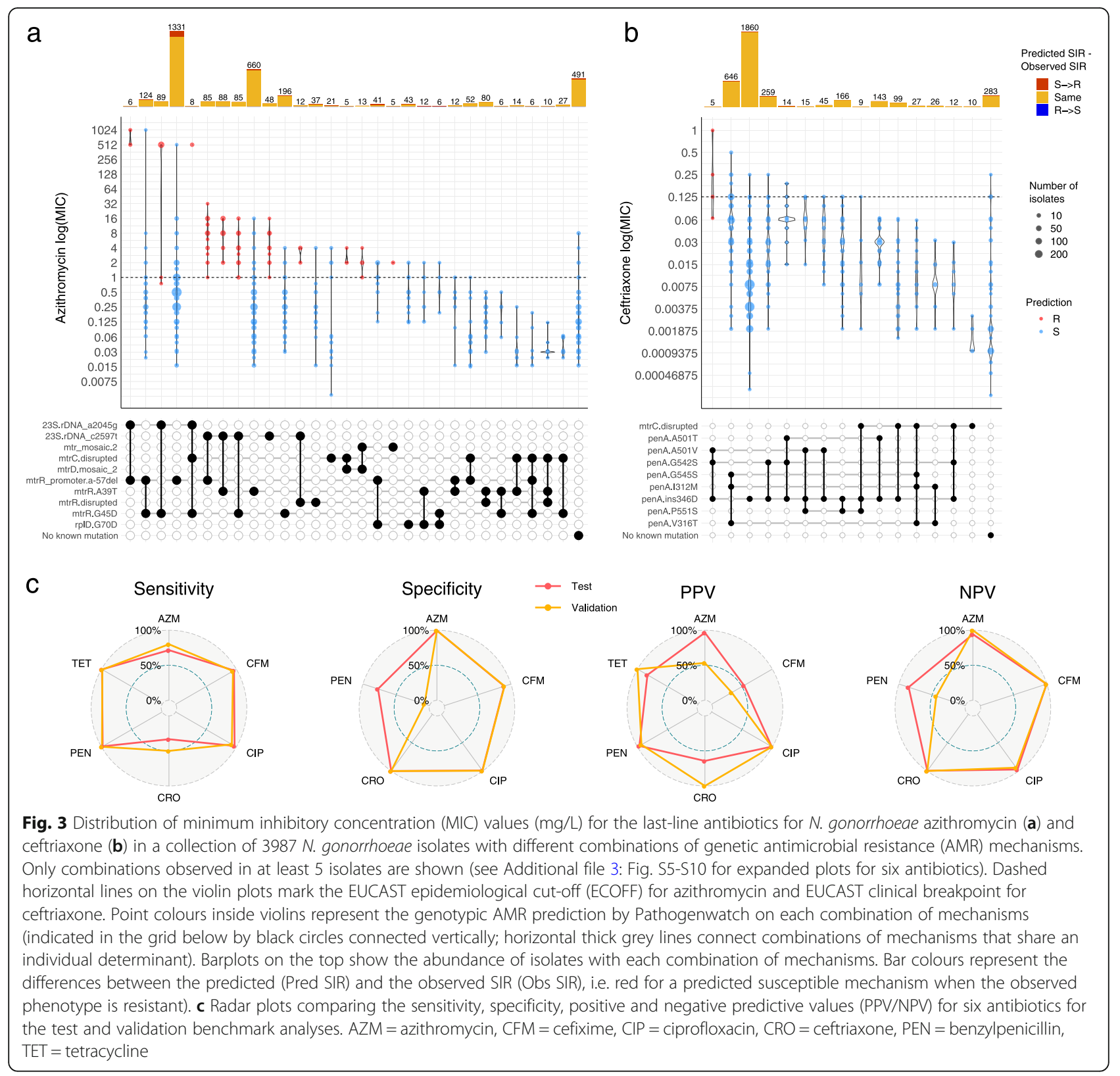

\section{Over 12,000 public genomes available}

Data for 11,461 isolates were successfully assembled and passed all quality cut-offs, resulting in 12,515 isolates after including the previously available Euro-GASP 2013 dataset [15]. New assemblies were uploaded and made public on Pathogenwatch, which now constitutes the largest repository of curated $N$. gonorrhoeae genomic data with associated metadata, typing and AMR information at the time of submission of this manuscript. Updated data spans 27 different publications $[18,54,59-62,64-66,68-71,117-131]$ and is organised into individual collections associated with the different studies (Additional file 1: Table S6). Available metadata was added for the genomes from these publications while basic metadata fields were kept for others (country, year/date and ENA project number).

We cross-checked that the main clusters found in the phylogenetic trees obtained after creating the public collections in Pathogenwatch were consistent with those observed in the trees in the corresponding publications. For example, recent works defined two major clusters of $N$. gonorrhoeae, termed Lineages $\mathrm{A}$ and $\mathrm{B}$, which were found to be consistent with the corresponding Pathogenwatch trees as exemplified for isolates from England in Town et al. [69] (Additional file 3: Fig. S11a). We were also able to differentiate the cefixime-resistant penA10- and penA34-carrying clones from Vietnam from Lan et al. [124] (Additional file 3: Fig. S11b) as well 
as the 10 major clusters defined in the $N$. gonorrhoeae population circulating in New York City (NYC) as described in Mortimer et al. [120] (Additional file 3: Fig. S11c). In the last case, we also liked to emphasise the usefulness of Microreact [37] as a parallel tool to Pathogenwatch for more complex visualisation purposes, such as showing the 10 major clusters in NYC as metadata blocks of different colours.

The $N$. gonorrhoeae public data available on Pathogenwatch spans nearly a century (1928-2018) and almost 70 different countries (Additional file 3: Fig. S12). However, sequencing efforts are unevenly distributed around the world, and over $90 \%$ of the published isolates were isolated in only 10 countries, headed by the UK $(N=$ $3476)$, the USA $(N=2774)$ and Australia $(N=2388)$
(Fig. 4, Additional file 1: Table S7). A total of 554 MLST, 1670 NG-MAST and 1769 NG-STAR STs were found in the whole dataset, from which a considerable number were new profiles caused by previously undetected alleles or new combinations of known alleles $(N=92$ new MLST STs, $N=769$ new NG-STAR STs and $N=$ 2289 isolates with new NG-MAST porB and/or tbpB alleles). These new alleles and profiles were submitted to the corresponding scheme servers.

Genomic studies are often biased towards AMR isolates, and this is reflected in the most abundant STs found for the three typing schemes within the public data. Isolates with MLST ST1901, ST9363 and ST7363, which contain resistance mechanisms to almost every antibiotic included in the study, represent over $25 \%$ of

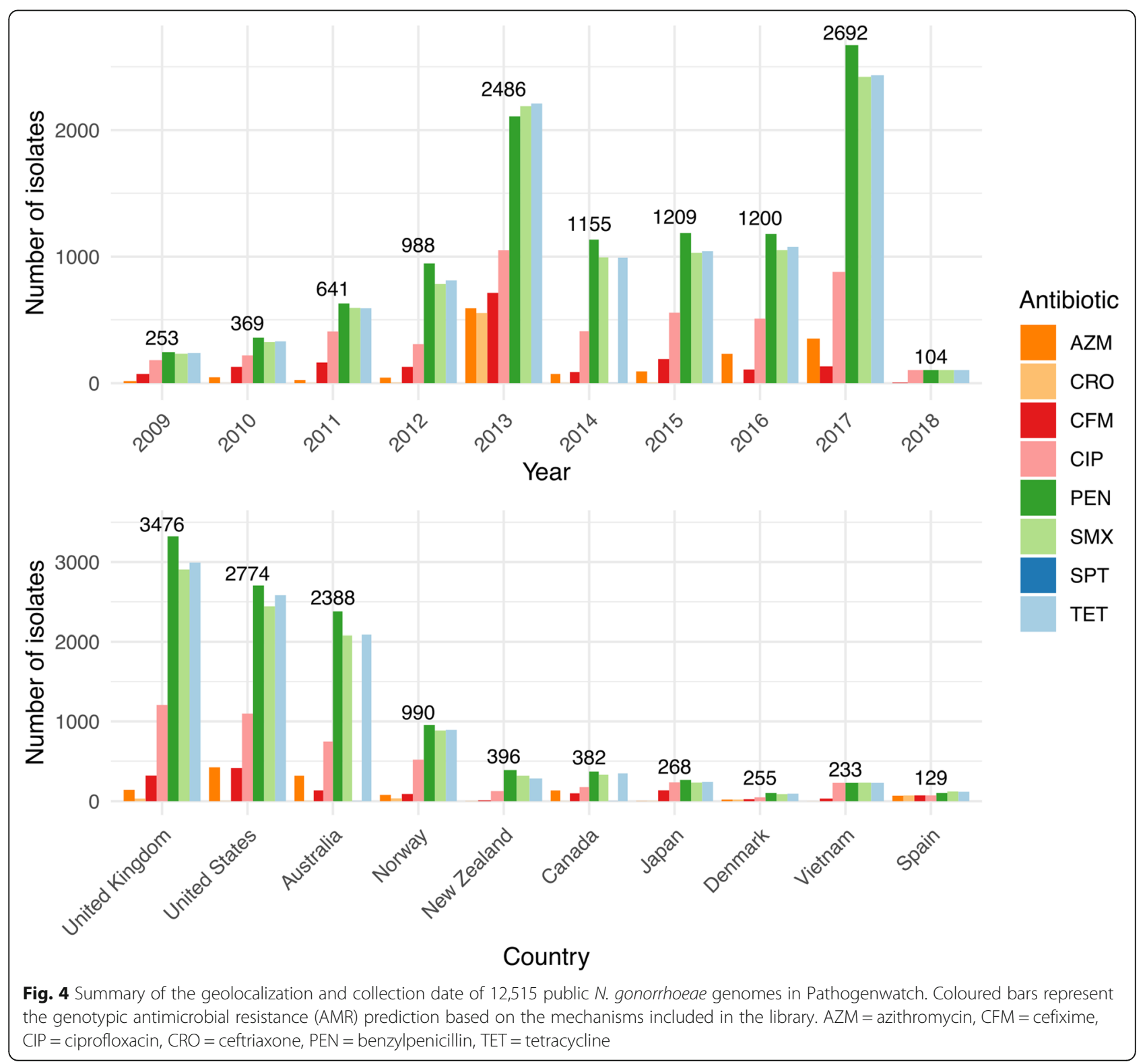




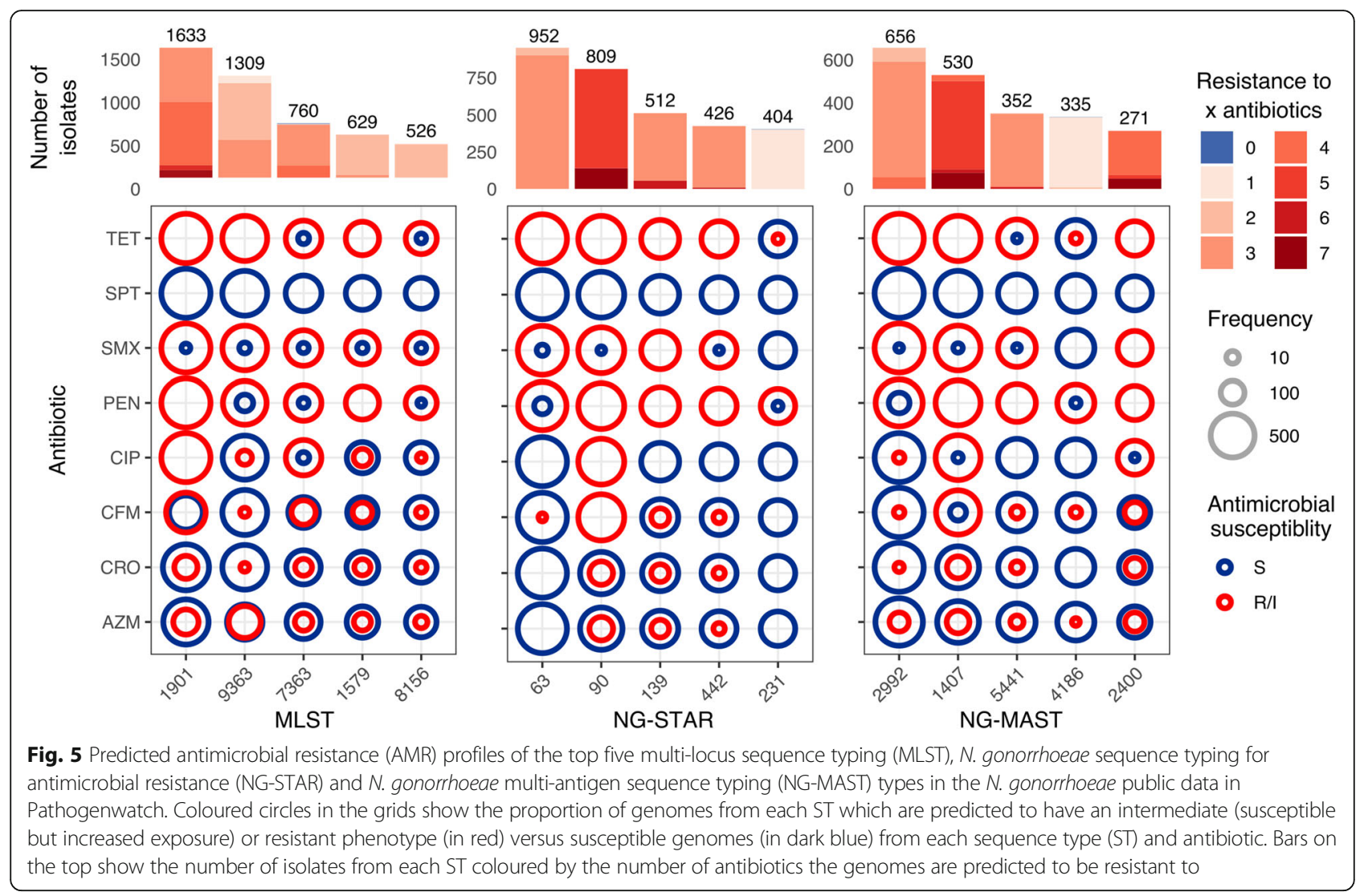

the data (Fig. 5). Isolates with MLST ST1901 and ST7363 are almost always associated with resistance to tetracycline, sulfonamides, benzylpenicillin and ciprofloxacin and nearly $50 \%$ of isolates from these two types harbour resistance mechanisms to cefixime. Ciprofloxacin resistance is not very widespread among ST9363 isolates, which are associated with azithromycin resistance in nearly $50 \%$ of the isolates of this ST (Fig. 5). NG-STAR ST63 (carrying the non-mosaic penA-2 allele, pen $A$ A517G and $m t r R$ A39T mutations as described in [52]) is the most represented in the dataset and carries resistance mechanisms to tetracycline, sulfonamides and benzylpenicillin, but is largely susceptible to spectinomycin, ciprofloxacin, the ESCs cefixime and ceftriaxone and azithromycin. NG-STAR ST90 isolates, conversely, are largely associated with resistance to cefixime, ciprofloxacin and benzylpenicillin as they carry the key resistance mutations in mosaic pen $A-34$, as well as in the $m t r R$ promoter, porB1b, ponA, $\operatorname{gyr} A$ and $\operatorname{parC}$ (as described in [52]). NGMAST ST1407 is commonly associated with MLST ST1901 and is the second most represented ST in the dataset following NG-MAST ST2992, which mainly harbours resistance to tetracycline, benzylpenicillin and sulfonamides (Fig. 5).

\section{Case study: global expansion of an $m$ tr mosaic-carrying clone}

The genetic mechanisms that have commonly been associated with an increased MIC of azithromycin in $N$. gonorrhoeae are two mutations in the 23S rRNA gene (A2045G and C2597T substitutions, in N. gonorrhoeae nomenclature) as well as mutations in $m$ trR and its promoter $[132,133]$. As described above, other mechanisms have also been recently discovered that increase the MIC of azithromycin (Table 2), such as mosaicism affecting the efflux pump-encoding $m \operatorname{tr} C D E$ genes and its repressor $m t r R$, mainly when the mosaic spans the $m t r R$ promoter region and $m \operatorname{trD}$ gene [23, 24]. Some studies have recently reported the local expansion of azithromycin-resistant $N$. gonorrhoeae lineages carrying an mtr mosaic in the USA [122, 123, 134] and Australia [118]. However, the extent of the dispersion of this mechanism to other parts of the world has not been studied yet. Here, using the public genomes of $N$. gonorrhoeae in Pathogenwatch, we have been able to explore this question.

A total of 1142 strains with genetic determinants of azithromycin resistance were selected in Pathogenwatch and combined with 395 genomes from a global 


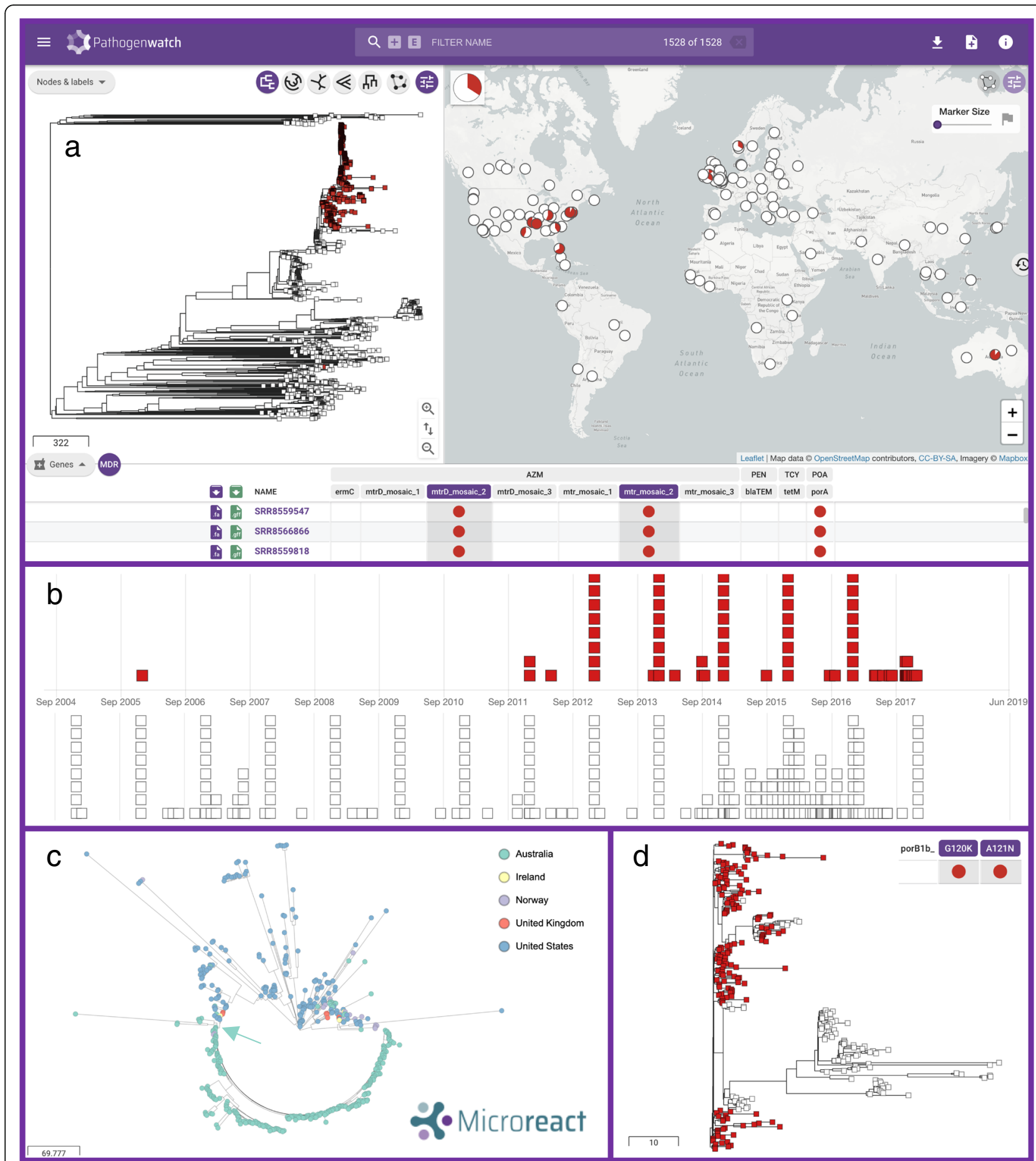

Fig. 6 N. gonorrhoeae genomes carrying genetic AMR mechanisms associated with azithromycin resistance were selected in Pathogenwatch $(n=$ 1142 ) and combined with genomes from a global collection $[65,112]$ (total $n=1528)$ for background contextualization. a Main layout of the combined collection, with an emerging lineage carrying an N. lactamica-like $m$ tr mosaic ('mtr_mosaic.2') spanning the $m t r R$ promoter and $m t r D$ marked in red in the tree and the map. b Timeline of the genomes carrying mtr mosaic 2 (in red) and other public genomes in the database without this genetic AMR mechanism. c Visualisation of the mtr mosaic 2-carrying lineage $(n=520)$ spreading in the USA and Australia (see legend) using Microreact. The arrow in turquoise colour marks the divergence of the Australian lineage, shown in more detail in $\mathbf{d}$ coloured by the presence (in red) or absence (in white) of the porB16 G120K and A121N mutations. The Pathogenwatch project of this case study can be explored in [135]. The contents of and boundaries in the map are the sole responsibility of Pathogenwatch and do not necessarily reflect the views or opinions of WHO or other Public Health Agency 
collection [65] for background contextualization (see Pathogenwatch project in [135]) (Fig. 6a). Five hundred seventy-one of the strains predicted to be resistant to azithromycin had some form of mosaic in the $m t r R$ promoter and/or $m t r D$ gene, of those described in Wadsworth et al. [23] (Table 2). These mosaics have been experimentally proven to increase MIC of azithromycin above $1 \mathrm{mg} / \mathrm{L}$, which is the EUCAST ECOFF value as well as the Clinical Laboratory and Standards Institute (CLSI) non-susceptibility breakpoint [23, 24]. One of the Neisseria lactamica-like mosaics, termed here 'mtr_mosaic.2', was by far the most extended, as it was found in 545 genomes spanning the $m t r R$ promoter and/or the $m$ trD gene, with 521 (95.6\%) of them spanning both regions. Twenty-five genomes contained a $N$. meningitidis-like mosaic $m t r R$ promoter and/or $m t r D$ gene (" $m t r_{-}$ mosaic.1') and in only 9 (36\%) of them the mosaic spanned both loci. The $N$. lactamica-like 'mtr_mosaic.3' was only found in isolate ERR855360 (GCGS834) from Los Angeles (USA, 2012), which is where the reference sequence for this mosaic was extracted from. Of the studies where these $m t r$ mosaic-carrying genomes were obtained from, only those from the USA and Australia specifically targeted and found this genetic determinant of resistance. The rest did not target this mosaic and some of them found strains with unexplained increased MICs of azithromycin [70, 121, 129], which could partly be explained by the presence of these $m$ tr mosaics.

We observed one main lineage with 520 genomes carrying the $N$. lactamica-like mosaic 2 in the $m t r R$ promoter and $m$ trD gene (Fig. 6a). Of those, only 3 and 8 isolates carried the 23S rDNA A2045G and C2597T mutations, respectively. Interestingly, the first strain in the database with this type of mosaic dates from 2006 [18]; however, it was not until the end of 2011-2012 when this lineage started to expand (Fig. 6b). Despite the genomic data contained in Pathogenwatch being biased to the amount of data sequenced and published from each country and year, we can easily infer that this lineage has spread across the world as we detect cases in Australia $(n=293)$ [118], the USA $(n=195)[18,120$, 122, 123], Norway $(n=19)$ [121], the UK $(n=11)$ [69, $119]$ and Ireland $(n=3)$ [129]. A strong association was found to the country of isolation (Fig. 6c), with a broad diversity of sublineages having spread across the USA (strains mostly isolated between 2012 and 2016). In contrast, an expansion of a particular clone, likely from a single main introduction, was observed to have occurred in Australia (strains isolated in 2017), followed by the further divergence of a subclone within the country which correlates with the loss of the porB1b G120K and A121N mutations (Fig. 6d), likely through a recombination event. Despite epidemiological data not being available for the Australian study [118], from their work we know that the clusters carrying an $m$ tr mosaic were mostly linked to transmission between men, although bridging among men who have sex with men (MSM) and heterosexual populations was also observed.

The results from our case study show that there is an emerging lineage of $N$. gonorrhoeae that has spread across the world and that is carrying a mosaic mtr that has been associated with low-to-medium resistance to azithromycin. This global lineage, as well as others that may emerge carrying this or other genetic AMR mechanisms, has to be closely monitored. For this purpose, an up-to-date genomic epidemiology tool such as Pathogenwatch, which includes a list of genetic AMR mechanisms approved by an expert group, is a great resource for the scientific community. At the moment, Pathogenwatch includes references for three types of mosaics in the $m t r R$ promoter and $m t r D$ genes that have been experimentally proven to increase MIC of azithromycin [23, 24], and the detection of these mosaics on new genomes respond to a set of similarity rules (see 'Availability of data and materials' section). However, we will keep the database updated with new experimentally confirmed reference sequences that may arise from further studies as it is still unclear whether all mosaics affecting the $m \operatorname{tr} C D E$ efflux pump decrease susceptibility to azithromycin.

\section{Discussion}

We present a public health-focussed $N$. gonorrhoeae framework at Pathogenwatch, an open access platform for genomic surveillance supported by an expert group that can be adapted to any public health or microbiology laboratory. Little bioinformatics expertise is required, and users can choose to either upload raw short-read data or assembled genomes. In both cases, the upload of high-quality data is encouraged in the form of qualitychecked reads and/or quality-checked assemblies. Recent benchmark analyses show particular recommendations for long-read or hybrid data [136] as well as short-readonly data $[40,137]$. On upload, several analyses are run on the genomes, and results for the three main typing schemes (MLST, NG-MAST and NG-STAR) as well as the detection of genetic determinants of AMR and a prediction of phenotypic resistance using these mechanisms can be obtained simultaneously. The library of AMR determinants contained in Pathogenwatch for N. gonorrhoeae has been revised and extended to include the latest mechanisms and epistatic interactions with experimental evidence of decreasing susceptibility or increasing resistance to at least one of eight antibiotics (Table 2). A test and validation benchmark analyses revealed sensitivity and/or specificity values $>90 \%$ for most of the tested antibiotics (Additional file 1: Table S3). Sensitivity values for the antimicrobials in the 
current dual treatment, azithromycin (80\%) and ceftriaxone $(50 \%)$, reflect the complexity of the resistance mechanisms for these antibiotics, for which we can only explain part of the observed phenotypic resistance. However, their specificity values were above 99\% (Additional file 1: Table S3), further strengthening the associations of the included AMR determinants in increasing MICs of these antibiotics. It remains essential to perform phenotypic susceptibility testing so we can detect inconsistencies between phenotypic and genotypic data that can lead to the identification and subsequent verification of novel or unknown resistance mechanisms. This will allow to continuously expand the list of genetic AMR mechanisms, and the AMR prediction from genomic data will further improve.

The continuous increase in reporting of $N$. gonorrhoeae AMR isolates worldwide led to a call for international collaborative action in 2017 to join efforts towards a global surveillance scheme. This was part of the WHO global health sector strategy on STIs (2016-2021), which set the goal of ending STI epidemics as a public health concern by year $2030[7,8]$. Several programmes are currently in place at different global, regional or national levels to monitor gonococcal AMR trends, emerging resistances and refine treatment guidelines and public health policies. This is the case of, for example, the WHO Global Gonococcal Antimicrobial Surveillance Programme (WHO GASP) $[7,8]$, the Euro-GASP in Europe [6, 15, 138], the Gonococcal Isolate Surveillance Project (GISP) in the USA [139], the Canadian Gonococcal Antimicrobial Surveillance Programme [140], the Australian Gonococcal Surveillance Programme (AGSP) [141] or the Gonococcal Resistance to Antimicrobials Surveillance Programme (GRASP) in England and Wales [142]. The WHO in collaboration with $\mathrm{CDC}$ has recently started an enhanced GASP (EGASP) [143] in some sentinel countries such as the Philippines and Thailand [144], aimed at collecting standardised and quality-assured epidemiological, clinical, microbiological and AMR data. On top of these programs, WHO launched the Global AMR Surveillance System (GLASS) in 2015 to foster national surveillance systems and enable standardised, comparable and validated AMR data on priority human bacterial pathogens [145]. Efforts are now underway to link WHO GASP to GLASS. However, gonococcal AMR surveillance is still suboptimal or even lacking in many locations, especially in LMICs, such as several parts of Asia, Central and Latin America, Eastern Europe and Africa, which worryingly have the greatest incidence of gonorrhoea [3]. LMICs often have access to antimicrobials without prescription, have limited access to an optimal treatment, lack the capacity needed to perform a laboratory diagnosis due to limited or nonexistent quality-assured laboratories, microbiological and bioinformatics expertise or training, insufficient availability and exorbitant prices of some reagents on top of a lack of funding, which altogether compromises infection control.

High-throughput sequencing approaches have proved invaluable over traditional molecular methods to identify AMR clones of bacterial pathogens, outbreaks, transmission networks and national and international spread among others [28, 29]. Genomic surveillance efforts to capture the local and international spread of $N$. gonorrhoeae have resulted in several publications within the last decade involving high-throughput sequence data of thousands of isolates from many locations across the world. The analysis of this data requires expertise, not always completely available, in bioinformatics, genomics, genetics, AMR, phylogenetics, epidemiology, etc. For lower-resourced settings, initiatives such as the NIHR Global Health Research Unit, Genomic Surveillance of Antimicrobial Resistance [146] are essential to build genomic surveillance capacity and provide the necessary microbiology and bioinformatics training for qualityassured genomic surveillance of AMR.

One of the strengths of genomic epidemiology is being able to compare new genomes with existing data from a broader geographical level, which provides additional information on, e.g. if new cases are part of a single clonal expansion or multiple introductions from outside a specific location. To support this, Pathogenwatch calculates phylogenetic trees from a set of genomes selected as collections. Currently, over 12,000 isolates of N. gonorrhoeae have been sequenced using high-throughput approaches and publicly deposited on the ENA linked to a scientific publication. We have quality-checked and assembled these data using a common pipeline and we made it available through Pathogenwatch, with the aim of representing as much genomic diversity of this pathogen as possible to serve as background for new analyses. These public genomes are associated with at least 27 different scientific publications and have been organised in Pathogenwatch as individual collections (Additional file 1: Table S6). The clustering of strains on the resulting phylogenetic reconstructions was found consistent with those in the original publications (some examples in Additional file 3: Fig. S11), while differences in branch lengths may be attributed to the usage of different reconstruction methods.

The power of Pathogenwatch to investigate questions of public health concern is reflected in a case study (Fig. 6). By selecting 1142 azithromycinresistant strains from the public data in Pathogenwatch in the context of a global collection [65], we observed one clone carrying a $N$. lactamica-like $m t r$ mosaic ('mtr_mosaic.2') in both the $m$ trR promoter and $m \operatorname{tr} D$ genes, likely resulting from the same recombination event. Strong geographical structure was 
found in these azithromycin-resistant strains, with isolates from the USA (mostly from 2012 to 2016) clearly differentiated from those from Australia (from 2017), which show a more clonal dispersion, likely from a single main introduction to the country followed by a rapid spread. Interestingly, a sublineage of this Australian mtr mosaic-carrying clone seems to have also diverged after losing the porB1b G120K and $\mathrm{D} 121 \mathrm{~N}$ mutations. It is important to note that the data from which these inferences were derived was gathered from surveillance-based studies and outbreak investigations, which may bias the observed global diversity of strains carrying this mosaic. Phenotypic susceptibility data for azithromycin or epidemiological information were not available for over half of these strains, thus impeding making further inferences. This reflects the need of improving the submission of anonymized epidemiological and antimicrobial susceptibility data of individual isolates, instead of aggregated data, to public repositories or as supplementary information of scientific publications, because this is where the public data in Pathogenwatch is coming from.

In this study, we have additionally gathered an advisory group of $N$. gonorrhoeae experts in different fields such as AMR, microbiology, genetics, genomics, epidemiology and public health who will consult and discuss current and future analytics to be included to address the global public health needs of the community. We suggest this strategy as a role model for other pathogens in this and other genomic surveillance platforms, so the end user, who may not have full computational experience in some cases, can be confident that the analytics and databases underlying this tool are appropriate and can have access to all the results provided by Pathogenwatch through uploading the data via a web browser. We are aware that this is a constantly moving field and analytics will be expanded and updated in the future. These updates will be discussed within the advisory group to make sure they are useful in the field and the way results are reported is of use to different profiles (microbiologists, epidemiologists, public health professionals, etc.). Future analytics that are under discussion include the automatic submission of new MLST, NG-STAR and NG-MAST STs and alleles to the corresponding servers, e.g. PubMLST [48] and the automatic submission of data to public archives such as the ENA. Interconnectivity and comparability of results with PubMLST is of particular interest, as this database has traditionally been the reference for Neisseria sequence typing and genomics and it is widely used by the $N$. gonorrhoeae community. Plasmid and tetM/blaTEM subtyping as recently described [147] will also be considered within the development roadmap of Pathogenwatch. Including a separate library to automatically screen targets of potential interest for vaccine design [148-150] as well as targets of new antibiotics currently in phase III clinical trials (i.e. zoliflodacin [151] or gepotidacin [152]) can also be an interesting addition to the scheme. Regarding AMR, new methods for phenotypic prediction using genetic data are continuously being reported [63, 153, 154], especially those based on machine learning algorithms [155], and will be considered for future versions of the platform. The prediction of MIC values or ranges instead of SIR categories will allow users to decide whether to use EUCAST [156] or CLSI [157] guidelines for categorisation.

\section{Conclusions}

In summary, we present a genomic surveillance platform adapted to $N$. gonorrhoeae, one of the main public health priorities compromising the control of AMR infections, where decisions on existing and updated databases and analytics as well as how results are reported will be discussed with an advisory board of experts in different public health areas. This will allow scientists from both higher- or lower-resourced settings with different capacities regarding high-throughput sequencing, bioinformatics and data interpretation, to be able to use a reproducible and quality-assured platform with analysed and contextualised genomic data resulting from the investigation of treatment failures, outbreaks, transmission chains and networks at different regional scales. This open access and reproducible platform (https://pathogen. watch/ngonorrhoeae) constitutes one step further into an international collaborative effort where countries can keep ownership of their data in line with national STI and AMR surveillance and control programs while aligning with global strategies for a joint action towards battling AMR N. gonorrhoeae.

\footnotetext{
Abbreviations

AGSP: Australian gonococcal surveillance programme; AMR: Antimicrobial resistance; AZM: Azithromycin; CDC: Centers for disease control and prevention; CFM: Cefixime; cgMLST: Core genome multi-locus sequence typing; CIP: Ciprofloxacin; CLSI: Clinical laboratory and standards institute; CRO: Ceftriaxone; ECOFF: Epidemiological cut-off; EGASP: Enhanced gonococcal antimicrobial surveillance programme; ENA: European nucleotide archive; ESCs: Extended-spectrum cephalosporins; EUCAST: European Committee on Antimicrobial Susceptibility Testing; Euro-GASP: European Gonococcal Antimicrobial Surveillance Programme; FN: False negative; FP: False positive; GASP: Gonococcal antimicrobial surveillance programme; GISP: Gonococcal isolate surveillance project; GRASP: Gonococcal resistance to antimicrobials surveillance programme; HIV: Human immunodeficiency virus; LMICs: Low- and middle-income countries; MIC: Minimum inhibitory concentration; MLST: Multi-locus sequence typing; MSM: Men who have sex with men; NG-MAST: N. gonorrhoeae multi-antigen sequence typing; NGSTAR: N. gonorrhoeae sequence typing for antimicrobial resistance; NPV: Negative predictive value; PEN: Benzylpenicillin; PPV: Positive predictive value; SNPS: Single-nucleotide polymorphisms; ST: Sequence type; STI: Sexually transmitted infection; TET: Tetracycline; TN: True negative;
} 
TP: True positive; UK: United Kingdom; WGS: Whole genome sequencing; WHO: World Health Organization

\section{Supplementary Information}

The online version contains supplementary material available at https://doi. org/10.1186/s13073-021-00858-2

Additional file 1: Table S1. List of studies included in the antimicrobial resistance benchmark analyses. Table S2. Point mutations and genes associated with antimicrobial resistance detected on the WHO 2016 reference panel. Table S3. Summary of the benchmark analysis. Table S4. List of genetic mechanisms detected on the test benchmark. Table S5. List of genetic mechanisms detected on the validation benchmark. Table S6. Public collections in N. gonorrhoeae Pathogenwatch. Table S7. Number of public N. gonorrhoeae genomes in Pathogenwatch by country.

Additional file 2: Results of the GHRU assembly pipeline on 12,192 public N. gonorrhoeae genomes.

Additional file 3: Figure S1. Main Pathogenwatch layout. Figure S2. Combination of Pathogenwatch collections. Figure S3. Genome reports and cgMLST cluster view. Figure S4. Mapping of short reads from WHO $U$ to the reference genome assembly. Figure S5. Minimum inhibitory concentration values for azithromycin in $\mathbf{N}$. gonorrhoeae isolates with different combinations of antimicrobial resistance genetic mechanisms.

Figure S6. Minimum inhibitory concentration values for ceftriaxone in $\mathrm{N}$. gonorrhoeae isolates with different combinations of antimicrobial resistance genetic mechanisms. Figure S7. Minimum inhibitory concentration values for cefixime in $\mathbf{N}$. gonorrhoeae isolates with different combinations of antimicrobial resistance genetic mechanisms. Figure S8. Minimum inhibitory concentration values for ciprofloxacin in $\mathrm{N}$. gonorrhoeae isolates with different combinations of antimicrobial resistance genetic mechanisms. Figure $\mathbf{5 9}$. Minimum inhibitory concentration values for tetracycline in $\mathbf{N}$. gonorrhoeae isolates with different combinations of antimicrobial resistance genetic mechanisms. Figure S10. Minimum inhibitory concentration values for benzylpenicillin in N. gonorrhoeae isolates with different combinations of antimicrobial resistance genetic mechanisms. Figure S11. Consistency of major N. gonorrhoeae population lineages and clusters observed in scientific publications with the Pathogenwatch phylogenetic trees.

Figure S12. Geographical distribution of public N. gonorrhoeae genomes in Pathogenwatch.

\section{Acknowledgements}

We would like to thank Gianfranco Spiteri (European Centre for Disease Prevention and Control, ECDC, Sweden) for his support and valuable insight and for being a member of the $N$. gonorrhoeae Pathogenwatch Scientific Steering Group together with coauthors LSB, DMA, MJC, YHG, IM, BHR, WMS, $\mathrm{KT}, \mathrm{TW}$ and MU.

\section{Authors' contributions}

DMA conceived the Pathogenwatch application. CY, RG, KA, BT, AU and DMA developed the Pathogenwatch application. LSB and DMA contributed to the conception and design of the work. CY and LSB generated, updated and benchmarked the N. gonorrhoeae AMR library. BT, CY, AU and LSB obtained, quality-checked and reassembled the raw data from the ENA. LSB revised the assembled data, obtained all metadata available from the corresponding scientific publications and created collections. LSB, CY and DMA analysed the data. LSB and DMA drafted the manuscript. LSB, DMA, CY, SA, KCM, TDM, DG, MJC, YHG, IM, BHR, WMS, KT, TW, SRH and MU contributed to the acquisition, technical and scientific interpretation and discussion of the data. LSB, DMA, MJC, YHG, IM, BHR, WMS, KT, TW and MU agreed to participate in the N. gonorrhoeae Pathogenwatch Scientific Steering Group before the preparation of this manuscript, and participated in virtual discussions. All authors read and approved the final manuscript.

\section{Funding}

Pathogenwatch is developed with support from Li Ka Shing Foundation (Big Data Institute, University of Oxford) and Wellcome (099202). At the time of preparation of this manuscript, LSB was supported by the Li Ka Shing
Foundation (Big Data Institute, University of Oxford) and the Centre for Genomic Pathogen Surveillance (CGPS, https://www.pathogensurveillance. net/). At the time of review and publication of this manuscript, LSB is funded by Plan GenT (CDEl-06/20-B), Conselleria de Sanitat Universal i Salut Pública, Generalitat Valenciana (Valencia, Spain). DMA is supported by the Li Ka Shing Foundation (Big Data Institute, University of Oxford) and the Centre for Genomic Pathogen Surveillance (CGPS). DMA and SA are supported by the National Institute for Health Research (UK) Global Health Research Unit on Genomic Surveillance of AMR (16_136_111). The department of MJC receives funding from the European Centre for Disease Prevention and Control and the National Institute for Health Research (Health Protection Research Unit) for gonococcal whole-genome sequencing. YHG is supported by the $\mathrm{NIH}$ / NIAID grants R01 Al132606 and R01 Al153521. KCM is supported by the NSF GRFP grant number DGE1745303. TDM is supported by the National Institute of Allergy and Infectious Diseases at the National Institutes of Health [1 F32 Al145157-01]. WMS is a recipient of a Senior Research Career Scientist Award from the Biomedical Laboratory Research and Development Service of the Department of Veterans. Work on antibiotic resistance in his laboratory is supported by NIH grants R37 Al-021150 and R01 Al-147609. The content of this article is solely the responsibility of the authors and does not necessarily represent the official views of the Department of Veterans Affairs, The National Institutes of Health or the United States Government. The findings and conclusions in this article are those of the author(s) and do not necessarily represent the official position of the Centers for Disease Control and Prevention. The WHO Collaborating Centre for Gonorrhoea and other STIs represented by DG and MU receives funding from the European Centre for Disease Prevention and Control and the World Health Organization. This publication made use of the Neisseria Multi-Locus Sequence Typing website (https://pubmlst.org/neisseria/) sited at the University of Oxford [48] and funded by Wellcome and European Union.

\section{Availability of data and materials}

The assemblies included in the current version of the $N$. gonorrhoeae Pathogenwatch scheme (https://pathogen.watch/ngonorrhoeae) and used for the AMR benchmark analyses were generated from raw sequencing data stored in the ENA. Project accession numbers are included in Additional File 1: Tables S1 and S6. The generated assemblies can be downloaded from Pathogenwatch. The AMR library can be accessed from https://gitlab.com/ cgps/pathogenwatch/amr-libraries/-/blob/master/485.toml [158]. The code to reproduce the figures and analyses in this manuscript can be found in https://gitlab.com/cgps/pathogenwatch/publications/-/tree/master/ ngonorrhoeae [159].

\section{Declarations}

Ethics approval and consent to participate

Not applicable.

\section{Consent for publication}

Not applicable.

\section{Competing interests}

The authors declare that they have no competing interests.

\section{Author details}

${ }^{1}$ Centre for Genomic Pathogen Surveillance, Big Data Institute, Nuffield Department of Medicine, University of Oxford, Oxford, Oxfordshire, UK. ${ }^{2}$ Genomics and Health Area, Foundation for the Promotion of Health and Biomedical Research in the Valencian Community (FISABIO-Public Health), Valencia, Spain. ${ }^{3}$ Centre for Genomic Pathogen Surveillance, Wellcome Sanger Institute, Wellcome Genome Campus, Cambridge, Cambridgeshire, UK. ${ }^{4}$ European Molecular Biology Lab, Heidelberg, Baden-Wuerttemberg, Germany. ${ }^{5}$ Department of Immunology and Infectious Diseases, Harvard T. H. Chan School of Public Health, Boston, MA, USA. ${ }^{6}$ World Health Organization Collaborating Centre for Gonorrhoea and Other STIS, Department of Laboratory Medicine, Faculty of Medicine and Health, Örebro University, Örebro, Sweden. ${ }^{7}$ National Infection Service, Public Health England, London, UK. ${ }^{8}$ Division of Infectious Diseases, Department of Medicine, Brigham and Women's Hospital, Harvard Medical School, Boston, MA, USA. ${ }^{9}$ National Microbiology Laboratory, Public Health Agency of Canada, Winnipeg, Manitoba, Canada. ${ }^{10}$ Division of STD prevention, National Center for HIV/ 
AIDS, Viral Hepatitis, STD and TB Prevention, Centers for Disease Control and Prevention, Atlanta, GA, USA. ${ }^{11}$ Department of Microbiology and Immunology and Emory Antibiotic Resistance Center, Emory University School of Medicine, Atlanta, GA, USA. ${ }^{12}$ Laboratories of Bacterial Pathogenesis, Veterans Affairs Medical Center, Decatur, GA, USA. ${ }^{13}$ Department of the Global HIV, Hepatitis and STI Programmes, World Health Organization, Geneva, Switzerland. ${ }^{14}$ Microbiotica, Biodata Innovation Centre, Cambridge, Cambridgeshire, UK.

Received: 3 July 2020 Accepted: 22 February 2021

Published online: 19 April 2021

\section{References}

1. World Health Organization (WHO). Global priority list of antibiotic-resistant bacteria to guide research, discovery, and development of new antibiotics. http://www.who.int/medicines/publications/WHO-PPL-Short_Summary_2 5Feb-ET_NM_WHO.pdf. 2017.

2. Centers for Disease Prevention and Control. U.S. Department of Health and Human Services, Atlanta, GA. Antibiotic Resistance Threats in the United States, 2019. https://www.cdc.gov/drugresistance/pdf/threats-report/2019-a r-threats-report-508.pdf. 2019

3. Rowley J, Vander Hoorn S, Korenromp E, Low N, Unemo M, Abu-Raddad LJ, et al. Chlamydia, gonorrhoea, trichomoniasis and syphilis: global prevalence and incidence estimates, 2016. Bull World Health Organ. 2019;97(8):548-62P.

4. World Health Organization (WHO). Report on global sexually transmitted infection surveillance 2018. http://apps.who.int/iris/bitstream/handle/1 0665/277258/9789241565691-eng.pdf?ua=1. 2019.

5. Unemo M, Shafer WM. Antimicrobial resistance in Neisseria gonorrhoeae in the 21st century: past, evolution, and future. Clin Microbiol Rev. 2014;27(3): 587-613.

6. Cole MJ, Spiteri G, Town K, Unemo M, Hoffmann S, Chisholm SA, et al. Risk factors for antimicrobial-resistant Neisseria gonorrhoeae in Europe. Sex Transm Dis. 2014;41(12):723-9.

7. Wi T, Lahra MM, Ndowa F, Bala M, Dillon JR, Ramon-Pardo P, et al. Antimicrobial resistance in Neisseria gonorrhoeae: global surveillance and a call for international collaborative action. PLoS Med. 2017;14(7):e1002344.

8. Unemo M, Lahra MM, Cole M, Galarza P, Ndowa F, Martin I, et al. World Health Organization Global Gonococcal Antimicrobial Surveillance Program (WHO GASP): review of new data and evidence to inform international collaborative actions and research efforts. Sex Health. 2019;16(5):412-25.

9. Fifer $\mathrm{H}$, Natarajan $\mathrm{U}$, Jones $\mathrm{L}$, Alexander $\mathrm{S}$, Hughes $\mathrm{G}$, Golparian $\mathrm{D}$, et al. Failure of dual antimicrobial therapy in treatment of gonorrhea. N Engl J Med. 2016;374(25):2504-6.

10. Jennison A, Whiley D, Lahra M, Graham R, Cole M, Hughes G, et al. Genetic relatedness of ceftriaxone-resistant and high-level azithromycin resistant Neisseria gonorrhoeae cases, United Kingdom and Australia, February to April 2018. Euro Surveill. 2019;24(8):1900118.

11. Lahra MM, Martin I, Demczuk W, Jennison AV, Lee KI, Nakayama SI, et al. Cooperative recognition of internationally disseminated ceftriaxone-resistant Neisseria gonorrhoeae Strain. Emerg Infect Dis. 2018;24(4):735-43.

12. Fifer H, Saunders J, Soni S, Sadiq ST, FitzGerald M. 2018 UK national guideline for the management of infection with Neisseria gonorrhoeae. Int J STD AIDS. 2020;31(1):4-15.

13. Deguchi T, Yasuda M, Asano M, Tada K, Iwata H, Komeda H, et al. DNA gyrase mutations in quinolone-resistant clinical isolates of Neisseria gonorrhoeae. Antimicrob Agents Chemother. 1995;39(2):561-3.

14. Tanaka M, Takahashi K, Saika T, Kobayashi I, Ueno T, Kumazawa J. Development of fluoroquinolone resistance and mutations involving GyrA and ParC proteins among Neisseria gonorrhoeae isolates in Japan. J Urol. 1998;159(6):2215-9.

15. Harris SR, Cole MJ, Spiteri G, Sanchez-Buso L, Golparian D, Jacobsson S, et al. Public health surveillance of multidrug-resistant clones of Neisseria gonorrhoeae in Europe: a genomic survey. Lancet Infect Dis. 2018;18(7):758-68.

16. Spratt BG. Hybrid penicillin-binding proteins in penicillin-resistant strains of Neisseria gonorrhoeae. Nature. 1988;332(6160):173-6.

17. Ohnishi M, Golparian D, Shimuta K, Saika T, Hoshina S, Iwasaku K, et al. Is Neisseria gonorrhoeae initiating a future era of untreatable gonorrhea?: detailed characterization of the first strain with high-level resistance to ceftriaxone. Antimicrob Agents Chemother. 2011;55(7):3538-45.

18. Grad YH, Harris SR, Kirkcaldy RD, Green AG, Marks DS, Bentley SD, et al. Genomic epidemiology of gonococcal resistance to extended spectrum cephalosporins, macrolides, and fluoroquinolones in the US, 2000-2013. J Infect Dis. 2016;214:1579-87.

19. Abrams AJ, Kirkcaldy RD, Pettus K, Fox JL, Kubin G, Trees DL. A case of decreased susceptibility to ceftriaxone in Neisseria gonorrhoeae in the absence of a mosaic penicillin-binding protein 2 (penA) allele. Sex Transm Dis. 2017;44(8):492-4.

20. Lindberg R, Fredlund $H$, Nicholas $R$, Unemo M. Neisseria gonorrhoeae isolates with reduced susceptibility to cefixime and ceftriaxone: association with genetic polymorphisms in penA, mtrR, porB1b, and ponA. Antimicrob Agents Chemother. 2007;51(6):2117-22.

21. Palace SG, Wang Y, Rubin DH, Welsh MA, Mortimer TD, Cole K, et al. RNA polymerase mutations cause cephalosporin resistance in clinical Neisseria gonorrhoeae isolates. Elife. 2020;9

22. Unemo M, Golparian D, Sanchez-Buso L, Grad Y, Jacobsson S, Ohnishi M, et al. The novel 2016 WHO Neisseria gonorrhoeae reference strains for global quality assurance of laboratory investigations: phenotypic, genetic and reference genome characterization. J Antimicrob Chemother. 2016;71(11):3096-108.

23. Wadsworth CB, Arnold BJ, Sater MRA, Grad YH. Azithromycin resistance through interspecific acquisition of an epistasis-dependent efflux pump component and transcriptional regulator in Neisseria gonorrhoeae. mBio. 2018;9(4):e01419-18.

24. Rouquette-Loughlin CE, Reimche JL, Balthazar JT, Dhulipala V, Gernert KM, Kersh EN, et al. Mechanistic basis for decreased antimicrobial susceptibility in a clinical isolate of Neisseria gonorrhoeae possessing a mosaic-like mtr efflux pump locus. mBio. 2018;9(6):e02281-18.

25. Ma KC, Mortimer TD, Duckett MA, Hicks AL, Wheeler NE, Sanchez-Buso L, et al. Increased power from conditional bacterial genome-wide association identifies macrolide resistance mutations in Neisseria gonorrhoeae. Nat Commun. 2020;11(1):5374.

26. Ma KC, Mortimer TD, Hicks AL, Wheeler NE, Sanchez-Buso L, Golparian $D$, et al. Adaptation to the cervical environment is associated with increased antibiotic susceptibility in Neisseria gonorrhoeae. Nat Commun. 2020;11(1):4126

27. Unemo M, Dillon JA. Review and international recommendation of methods for typing Neisseria gonorrhoeae isolates and their implications for improved knowledge of gonococcal epidemiology, treatment, and biology. Clin Microbiol Rev. 2011;24(3):447-58.

28. Loman NJ, Pallen MJ. Twenty years of bacterial genome sequencing. Nat Rev Microbiol. 2015;13(12):787-94.

29. Balloux F, Bronstad Brynildsrud O, van Dorp L, Shaw LP, Chen H, Harris KA, et al. From theory to practice: translating whole-genome sequencing (WGS) into the clinic. Trends Microbiol. 2018;26(12):1035-48.

30. Boolchandani M, D'Souza AW, Dantas G. Sequencing-based methods and resources to study antimicrobial resistance. Nat Rev Genet. 2019; 20(6):356-70

31. Hendriksen RS, Bortolaia V, Tate H, Tyson GH, Aarestrup FM, McDermott PF. Using genomics to track global antimicrobial resistance. Front Public Health. 2019;7:242.

32. React JavaScript library. https://reactjs.org/. Accessed 24 Nov 2020.

33. Material Design Lite. https://getmdl.io. Accessed 24 Nov 2020.

34. Phylocanvas. http://phylocanvas.org. Accessed 24 Nov 2020.

35. Leaflet. https://leafletjs.com/. Accessed 24 Nov 2020.

36. Sigma JavaScript library. http://sigmajs.org/. Accessed 24 Nov 2020.

37. Argimon S, Abudahab K, Goater RJ, Fedosejev A, Bhai J, Glasner C, et al. Microreact: visualizing and sharing data for genomic epidemiology and phylogeography. Microb Genom. 2016;2(11):e000093.

38. Page AJ, Cummins CA, Hunt M, Wong VK, Reuter S, Holden MT, et al. Roary: rapid large-scale prokaryote pan genome analysis. Bioinformatics. 2015; 31(22):3691-3.

39. Centre for Genomic Pathogen Surveillance (CGPS). Pathogenwatch technical descriptions. Core assignment. https://cgps.gitbook.io/pathogenwa tch/technical-descriptions/core-genome-tree/core-assignment (2020).

40. Bankevich A, Nurk S, Antipov D, Gurevich AA, Dvorkin M, Kulikov AS, et al. SPAdes: a new genome assembly algorithm and its applications to singlecell sequencing. J Comput Biol. 2012;19(5):455-77.

41. Centre for Genomic Pathogen Surveillance (CGPS). Pathogenwatch technical descriptions. Short read assembly. https://cgps.gitbook.io/pa thogenwatch/technical-descriptions/short-read-assembly (2020).

42. CGPS. Pathogenwatch technical descriptions. Speciator. https://cgps. gitbook.io/pathogenwatch/technical-descriptions/species-assignment/ speciator (2020). 
43. Centre for Genomic Pathogen Surveillance (CGPS). Pathogenwatch technical descriptions. Core filter. https:/cgps.gitbook.io/pathogenwatch/ technical-descriptions/core-genome-tree/core-filter (2020).

44. Centre for Genomic Pathogen Surveillance (CGPS). Pathogenwatch technical descriptions. Tree construction. https://cgps.gitbook.io/pa thogenwatch/technical-descriptions/core-genome-tree/treeconstruction (2020).

45. Bennett JS, Jolley KA, Sparling PF, Saunders NJ, Hart CA, Feavers IM, et al. Species status of Neisseria gonorrhoeae: evolutionary and epidemiological inferences from multilocus sequence typing. BMC Biol. 2007;5:35.

46. Harrison OB, Cehovin A, Skett J, Jolley KA, Massari P, Genco CA, et al. Neisseria gonorrhoeae population genomics: use of the gonococcal core genome to improve surveillance of antimicrobial resistance. J Infect Dis. 2020;222(11):1816-25.

47. Public databases for molecular typing and microbial genome diversity (PubMLST). Neisseria Multi-Locus Sequence Typing (MLST) website. https:// pubmlst.org/neisseria/. Accessed 24 Nov 2020.

48. Jolley KA, Bray JE, Maiden MCJ. Open-access bacterial population genomics: BIGSdb software, the PubMLST.org website and their applications. Wellcome Open Res. 2018;3:124.

49. Martin IM, Ison CA, Aanensen DM, Fenton KA, Spratt BG. Rapid sequencebased identification of gonococcal transmission clusters in a large metropolitan area. J Infect Dis. 2004;189(8):1497-505.

50. Neisseria gonorrhoeae Multi-Antigen Sequence Typing (NG-MAST) database. http://www.ng-mast.net/. Accessed 24 Nov 2020.

51. Demczuk W, Sidhu S, Unemo M, Whiley DM, Allen VG, Dillon JR, et al. Neisseria gonorrhoeae sequence typing for antimicrobial resistance, a novel antimicrobial resistance multilocus typing scheme for tracking global dissemination of N. gonorrhoeae strains. J Clin Microbiol. 2017; 55(5):1454-68

52. Neisseria gonorrhoeae sequence typing for antimicrobial resistance (NGSTAR) database. https://ngstar.canada.ca/. Accessed 24 Nov 2020.

53. Page A, Taylor B, Keane J. Multilocus sequence typing by blast from de novo assemblies against PubMLST. J Open Source Softw. 2016;1(8):118.

54. Kwong JC, Gonçalves da Silva A, Dyet K, Williamson DA, Stinear TP, Howden $\mathrm{BP}$, et al. NGMASTER: in silico multi-antigen sequence typing for Neisseria gonorrhoeae. Microb Genom. 2016;2(8):e000076.

55. Centre for Genomic Pathogen Surveillance (CGPS). Pathogenwatch technical descriptions. MLST. https://cgps.gitbook.io/pathogenwatch/ technical-descriptions/typing-methods/mlst (2020).

56. Centre for Genomic Pathogen Surveillance (CGPS). Pathogenwatch technical descriptions. cgMLST clustering. https://cgps.gitbook.io/pa thogenwatch/technical-descriptions/cgmlst-clusters (2020).

57. Sibson R. SLINK: an optimally efficient algorithm for the single-link cluster method. Comput J. 1973;16(1):30-4.

58. Centre for Genomic Pathogen Surveillance (CGPS). Pathogenwatch technical descriptions. Pathogenwatch AMR. https://cgps.gitbook.io/pa thogenwatch/technical-descriptions/antimicrobial-resistance-prediction/ pw-amr (2020).

59. Chisholm SA, Wilson J, Alexander S, Tripodo F, Al-Shahib A, Schaefer U, et al. An outbreak of high-level azithromycin resistant Neisseria gonorrhoeae in England. Sex Transm Infect 2015;92(5):365-7.

60. Golparian D, Harris SR, Sanchez-Buso L, Hoffmann S, Shafer WM, Bentley SD, et al. Genomic evolution of Neisseria gonorrhoeae since the preantibiotic era (1928-2013): antimicrobial use/misuse selects for resistance and drives evolution. BMC Genomics. 2020;21(1):116.

61. Demczuk W, Lynch T, Martin I, Van Domselaar G, Graham M, Bharat A, et al. Whole-genome phylogenomic heterogeneity of Neisseria gonorrhoeae isolates with decreased cephalosporin susceptibility collected in Canada between 1989 and 2013. J Clin Microbiol. 2015;53(1):191-200.

62. Demczuk W, Martin I, Peterson S, Bharat A, Van Domselaar G, Graham M, et al. Genomic epidemiology and molecular resistance mechanisms of azithromycin-resistant Neisseria gonorrhoeae in Canada from 1997 to 2014. J Clin Microbiol. 2016;54(5):1304-13.

63. Eyre DW, De Silva D, Cole K, Peters J, Cole MJ, Grad YH, et al. WGS to predict antibiotic MICs for Neisseria gonorrhoeae. J Antimicrob Chemother. 2017;72(7):1937-47.

64. Fifer H, Cole M, Hughes G, Padfield S, Smolarchuk C, Woodford N, et al. Sustained transmission of high-level azithromycin-resistant Neisseria gonorrhoeae in England: an observational study. Lancet Infect Dis. 2018; 18(5):573-81.
65. Sanchez-Buso L, Golparian D, Corander J, Grad YH, Ohnishi M, Flemming R, et al. The impact of antimicrobials on gonococcal evolution. Nat Microbiol. 2019:4:1941-50

66. Grad YH, Kirkcaldy RD, Trees D, Dordel J, Harris SR, Goldstein E, et al. Genomic epidemiology of Neisseria gonorrhoeae with reduced susceptibility to cefixime in the USA: a retrospective observational study. Lancet Infect Dis. 2014;14(3):220-6.

67. Jacobsson S, Golparian D, Cole M, Spiteri G, Martin I, Bergheim T, et al. WGS analysis and molecular resistance mechanisms of azithromycin-resistant (MIC > 2 mg/L) Neisseria gonorrhoeae isolates in Europe from 2009 to 2014. J Antimicrob Chemother. 2016;71(11):3109-16.

68. Lee RS, Seemann T, Heffernan H, Kwong JC. Goncalves da Silva a, Carter GP, et al. genomic epidemiology and antimicrobial resistance of Neisseria gonorrhoeae in New Zealand. J Antimicrob Chemother. 2018; 73(2):353-64.

69. Town K, Harris S, Sanchez-Buso L, Cole MJ, Pitt R, Fifer H, et al. Genomic and phenotypic variability in Neisseria gonorrhoeae antimicrobial susceptibility, England. Emerg Infect Dis. 2020;26(3):505-15.

70. Yahara K, Nakayama SI, Shimuta K, Lee Kl, Morita M, Kawahata T, et al. Genomic surveillance of Neisseria gonorrhoeae to investigate the distribution and evolution of antimicrobial-resistance determinants and lineages. Microb Genom. 2018;4(8):e000205.

71. Kwong JC, Chow EPF, Stevens K, Stinear TP, Seemann T, Fairley CK, et al. Whole-genome sequencing reveals transmission of gonococcal antibiotic resistance among men who have sex with men: an observational study. Sex Transm Infect. 2018;94(2):151-7.

72. European Committee on Antimicrobial Susceptibility Testing (EUCAST). Breakpoint tables for interpretation of MICs and zone diameters. Version 9.0. https://www.eucast.org/fileadmin/src/media/PDFs/EUCAST_files/Breakpoint_ tables/v_9.0_Breakpoint_Tables.pdf. Accessed 24 November 2020.

73. Stevenson M, Nunes T, Heuer C, Marshall J, Sanchez J, Thornton R, et al. epiR: Tools for the Analysis of Epidemiological Data. R package version 1.014. https://CRAN.R-project.org/package=epiR. 2020.

74. Carver T, Harris SR, Berriman M, Parkhill J, McQuillan JA. Artemis: an integrated platform for visualization and analysis of high-throughput sequence-based experimental data. Bioinformatics. 2012;28(4):464-9.

75. Chisholm SA, Dave J, Ison CA. High-level azithromycin resistance occurs in Neisseria gonorrhoeae as a result of a single point mutation in the $23 \mathrm{~S}$ rRNA genes. Antimicrob Agents Chemother. 2010;54(9):3812-6.

76. Underwood A. Gobal Health Research Unit (GHRU) assembly pipeline. Gitlab. https://gitlab.com/cgps/ghru/pipelines/assembly (2020).

77. Andrews S. FastQC: a quality control tool for high throughput sequence data. https://www.bioinformatics.babraham.ac.uk/projects/fastqc/. (2010)

78. Bolger AM, Lohse M, Usadel B. Trimmomatic: a flexible trimmer for Illumina sequence data. Bioinformatics. 2014:30(15):2114-20.

79. Song L, Florea L, Langmead B. Lighter: fast and memory-efficient sequencing error correction without counting. Genome Biol. 2014 15(11):509.

80. Low AJ, Koziol AG, Manninger PA, Blais B, Carrillo CD. ConFindr: rapid detection of intraspecies and cross-species contamination in bacterial whole-genome sequence data. PeerJ. 2019;7:e6995.

81. Ondov BD, Treangen TJ, Melsted P, Mallonee AB, Bergman NH, Koren S, et al. Mash: fast genome and metagenome distance estimation using MinHash. Genome Biol. 2016;17(1):132.

82. Li H. Seqtk. Github. https://github.com/lh3/seqtk (2020).

83. Magoc T, Salzberg SL. FLASH: fast length adjustment of short reads to improve genome assemblies. Bioinformatics. 2011;27(21):2957-63.

84. Gurevich A, Saveliev V, Vyahhi N, Tesler G. QUAST: quality assessment tool for genome assemblies. Bioinformatics. 2013;29(8):1072-5.

85. Underwood A. Bactinspector. Gitlab. https://gitlab.com/antunderwood/ba ctinspector (2020).

86. Underwood A. Qualifyr. Gitlab. https://gitlab.com/cgps/qualifyr (2020).

87. European Nucleotide Archive (ENA) API portal. https://www.ebi.ac.uk/ena/ portal/api/. Accessed 24 Nov 2020.

88. Ng LK, Martin I, Liu G, Bryden L. Mutation in $23 \mathrm{~S}$ rRNA associated with macrolide resistance in Neisseria gonorrhoeae. Antimicrob Agents Chemother. 2002;46(9):3020-5.

89. Roberts MC, Chung WO, Roe D, Xia M, Marquez C, Borthagaray G, et al. Erythromycin-resistant Neisseria gonorrhoeae and oral commensal Neisseria spp. carry known rRNA methylase genes. Antimicrob Agents Chemother. 1999;43(6):1367-72. 
90. Cousin S Jr, Whittington WL, Roberts MC. Acquired macrolide resistance genes in pathogenic Neisseria spp. isolated between 1940 and 1987. Antimicrob Agents Chemother. 2003;47(12):3877-80.

91. Luna VA, Cousin S Jr, Whittington WL, Roberts MC. Identification of the conjugative mef gene in clinical Acinetobacter junii and Neisseria gonorrhoeae isolates. Antimicrob Agents Chemother. 2000;44(9):2503-6.

92. Rouquette-Loughlin CE, Balthazar JT, Shafer WM. Characterization of the MacA-MacB efflux system in Neisseria gonorrhoeae. J Antimicrob Chemother. 2005;56(5):856-60.

93. Veal WL, Nicholas RA, Shafer WM. Overexpression of the MtrC-MtrD-MtrE efflux pump due to an mtrR mutation is required for chromosomally mediated penicillin resistance in Neisseria gonorrhoeae. J Bacteriol. 2002; 184(20):5619-24

94. Cousin SL Jr, Whittington WL, Roberts MC. Acquired macrolide resistance genes and the 1 bp deletion in the mtrR promoter in Neisseria gonorrhoeae. J Antimicrob Chemother. 2003;51(1):131-3.

95. Warner DM, Shafer WM, Jerse AE. Clinically relevant mutations that cause derepression of the Neisseria gonorrhoeae MtrC-MtrD-MtrE efflux pump system confer different levels of antimicrobial resistance and in vivo fitness. Mol Microbiol. 2008;70(2):462-78.

96. Shafer WM, Balthazar JT, Hagman KE, Morse SA. Missense mutations that alter the DNA-binding domain of the MtrR protein occur frequently in rectal isolates of Neisseria gonorrhoeae that are resistant to faecal lipids. Microbiology. 1995;141(Pt 4):907-11.

97. Tomberg J, Unemo M, Davies C, Nicholas RA. Molecular and structural analysis of mosaic variants of penicillin-binding protein 2 conferring decreased susceptibility to expanded-spectrum cephalosporins in Neisseria gonorrhoeae: role of epistatic mutations. Biochemistry. 2010; 49(37):8062-70

98. Unemo M, Golparian D, Nicholas R, Ohnishi M, Gallay A, Sednaoui P. Highlevel cefixime- and ceftriaxone-resistant Neisseria gonorrhoeae in France: novel penA mosaic allele in a successful international clone causes treatment failure. Antimicrob Agents Chemother. 2012;56(3):1273-80.

99. Belland RJ, Morrison SG, Ison C, Huang WM. Neisseria gonorrhoeae acquires mutations in analogous regions of gyrA and parC in fluoroquinoloneresistant isolates. Mol Microbiol. 1994;14(2):371-80.

100. Tomberg J, Unemo M, Ohnishi M, Davies C, Nicholas RA. Identification of amino acids conferring high-level resistance to expanded-spectrum cephalosporins in the penA gene from Neisseria gonorrhoeae strain $\mathrm{H} 041$. Antimicrob Agents Chemother. 2013;57(7):3029-36.

101. Rouquette-Loughlin C, Dunham SA, Kuhn M, Balthazar JT, Shafer WM. The NorM efflux pump of Neisseria gonorrhoeae and Neisseria meningitidis recognizes antimicrobial cationic compounds. J Bacteriol. 2003;185(3):1101-6.

102. Lindback E, Rahman M, Jalal S, Wretlind B. Mutations in gyrA, gyrB, parC, and parE in quinolone-resistant strains of Neisseria gonorrhoeae. APMIS. 2002;110(9):651-7.

103. Hu M, Nandi S, Davies C, Nicholas RA. High-level chromosomally mediated tetracycline resistance in Neisseria gonorrhoeae results from a point mutation in the rps J gene encoding ribosomal protein S10 in combination with the mtrR and penB resistance determinants. Antimicrob Agents Chemother. 2005:49(10):4327-34.

104. Morse SA, Johnson SR, Biddle JW, Roberts MC. High-level tetracycline resistance in Neisseria gonorrhoeae is result of acquisition of streptococcal tetM determinant. Antimicrob Agents Chemother. 1986; 30(5):664-70.

105. Ashford WA, Golash RG, Hemming VG. Penicillinase-producing Neisseria gonorrhoeae. Lancet. 1976;2(7987):657-8.

106. Ropp PA, Hu M, Olesky M, Nicholas RA. Mutations in ponA, the gene encoding penicillin-binding protein 1, and a novel locus, penC, are required for high-level chromosomally mediated penicillin resistance in Neisseria gonorrhoeae. Antimicrob Agents Chemother. 2002;46(3):769-77.

107. Olesky M, Hobbs M, Nicholas RA. Identification and analysis of amino acid mutations in porin IB that mediate intermediate-level resistance to penicillin and tetracycline in Neisseria gonorrhoeae. Antimicrob Agents Chemother 2002:46(9):2811-20.

108. Bilgin N, Richter AA, Ehrenberg M, Dahlberg AE, Kurland CG. Ribosomal RNA and protein mutants resistant to spectinomycin. EMBO J. 1990;9(3):735-9.

109. Unemo M, Golparian D, Skogen V, Olsen AO, Moi H, Syversen G, et al. Neisseria gonorrhoeae strain with high-level resistance to spectinomycin due to a novel resistance mechanism (mutated ribosomal protein S5) verified in Norway. Antimicrob Agents Chemother. 2013;57(2):1057-61.
110. Fiebelkorn KR, Crawford SA, Jorgensen JH. Mutations in folP associated with elevated sulfonamide MICs for Neisseria meningitidis clinical isolates from five continents. Antimicrob Agents Chemother. 2005;49(2):536-40.

111. Zhao S, Duncan M, Tomberg J, Davies C, Unemo M, Nicholas RA. Genetics of chromosomally mediated intermediate resistance to ceftriaxone and cefixime in Neisseria gonorrhoeae. Antimicrob Agents Chemother. 2009;53(9):3744-51.

112. Centre for Genomic Pathogen Surveillance (CGPS). Pathogenwatch collection. Sánchez-Busó et al. (2019). https://pathogen.watch/collection/ 9stz0m94hin7-sanchez-buso-et-al-2019 (2020).

113. Sánchez-Busó L. N. gonorrhoeae Pathogenwatch video demo. https://vimeo. com/434706201. 2020

114. Ison CA, Golparian D, Saunders P, Chisholm S, Unemo M. Evolution of Neisseria gonorrhoeae is a continuing challenge for molecular detection of gonorrhoea: false negative gonococcal porA mutants are spreading internationally. Sex Transm Infect. 2013:89(3):197-201.

115. Kersh EN, Allen V, Ransom E, Schmerer M, Cyr S, Workowski K, et al. Rationale for a Neisseria gonorrhoeae susceptible-only interpretive breakpoint for azithromycin. Clin Infect Dis. 2020;70(5):798-804.

116. Cole MJ, Tan W, Fifer H, Brittain C, Duley L, Hepburn T, et al. Gentamicin, azithromycin and ceftriaxone in the treatment of gonorrhoea: the relationship between antibiotic MIC and clinical outcome. J Antimicrob Chemother. 2020;75(2):449-57.

117. Town K, Field N, Harris SR, Sanchez-Buso L, Cole MJ, Pitt R, et al. Phylogenomic analysis of Neisseria gonorrhoeae transmission to assess sexual mixing and HIV transmission risk in England: a cross-sectional, observational, whole-genome sequencing study. Lancet Infect Dis. 2020; 20(4):478-86.

118. Williamson DA, Chow EPF, Gorrie CL, Seemann T, Ingle DJ, Higgins N, et al. Bridging of Neisseria gonorrhoeae lineages across sexual networks in the HIV pre-exposure prophylaxis era. Nat Commun. 2019;10(1):3988.

119. De Silva D, Peters J, Cole K, Cole MJ, Cresswell F, Dean G, et al. Wholegenome sequencing to determine transmission of Neisseria gonorrhoeae: an observational study. Lancet Infect Dis. 2016;16(11):1295-303.

120. Mortimer TD, Pathela P, Crawley A, Rakeman JL, Lin Y, Harris SR, et al. The distribution and spread of susceptible and resistant Neisseria gonorrhoeae across demographic groups in a major metropolitan center. Clin Infect Dis. 2020;ciaa1229.

121. Alfsnes K, Eldholm V, Olsen AO, Brynildsrud OB, Bohlin J, Steinbakk $M$, et al. Genomic epidemiology and population structure of Neisseria gonorrhoeae in Norway, 2016-2017. Microb Genom. 2020;6(4):e000359.

122. Thomas JC, Seby S, Abrams AJ, Cartee J, Lucking S, Vidyaprakash E, et al. Evidence of recent genomic evolution in gonococcal strains with decreased susceptibility to cephalosporins or azithromycin in the United States, 20142016. J Infect Dis. 2019;220(2):294-305.

123. Schmerer MW, Abrams AJ, Seby S, Thomas JC, Cartee J, Lucking S, et al. Genomic characterization of Neisseria gonorrhoeae strains from 2016 U.S Sentinel surveillance displaying reduced susceptibility to azithromycin. Antimicrob Agents Chemother. 2020;64(5):e02420-19.

124. Lan PT, Golparian D, Ringlander J, Van Hung L, Van Thuong N, Unemo M. Genomic analysis and antimicrobial resistance of Neisseria gonorrhoeae isolates from Vietnam in 2011 and 2015-16. J Antimicrob Chemother. 2020; 75(6):1432-8.

125. Didelot X, Dordel J, Whittles LK, Collins C, Bilek N, Bishop CJ, et al. Genomic analysis and comparison of two gonorrhea outbreaks. MBio. 2016;7(3): e00525-16.

126. Osnes MN, Didelot X, Korne-Elenbaas J, Alfsnes K, Brynildsrud OB, Syversen $G$, et al. Sudden emergence of a Neisseria gonorrhoeae clade with reduced susceptibility to extended-spectrum cephalosporins, Norway. Microb Genom. 2020;6(12):e000480.

127. Cehovin A, Harrison OB, Lewis SB, Ward PN, Ngetsa C, Graham SM, et al. Identification of novel Neisseria gonorrhoeae lineages harboring resistance plasmids in Coastal Kenya. J Infect Dis. 2018;218(5):801-8.

128. Buckley C, Forde BM, Trembizki E, Lahra MM, Beatson SA, Whiley DM. Use of whole genome sequencing to investigate an increase in Neisseria gonorrhoeae infection among women in urban areas of Australia. Sci Rep. 2018;8(1):1503.

129. Ryan L, Golparian D, Fennelly N, Rose L, Walsh P, Lawlor B, et al. Antimicrobial resistance and molecular epidemiology using whole-genome sequencing of Neisseria gonorrhoeae in Ireland, 2014-2016: focus on extended-spectrum cephalosporins and azithromycin. Eur J Clin Microbiol Infect Dis. 2018;37(9):1661-72. 
130. Wind CM, de Vries E, van der Loeff MF S, van Rooijen MS, van Dam AP, WHB D, et al. Decreased azithromycin susceptibility of Neisseria gonorrhoeae isolates in patients recently treated with azithromycin. Clin Infect Dis. 2017; 65(1):37-45.

131. Ezewudo MN, Joseph SJ, Castillo-Ramirez S, Dean D, Del Rio C, Didelot $X$, et al. Population structure of Neisseria gonorrhoeae based on whole genome data and its relationship with antibiotic resistance. PeerJ. 2015;3:e806.

132. Unemo M, Golparian D, Eyre DW. Antimicrobial resistance in Neisseria gonorrhoeae and treatment of gonorrhea. Methods Mol Biol. 1997; 2019:37-58.

133. Mortimer TD, Grad YH. Applications of genomics to slow the spread of multidrug-resistant Neisseria gonorrhoeae. Ann N Y Acad Sci. 2019;1435(1): 93-109.

134. Gernert KM, Seby S, Schmerer MW, Thomas JC, Pham CD, Cyr SS, et al. Azithromycin susceptibility of Neisseria gonorrhoeae in the USA in 2017: a genomic analysis of surveillance data. Lancet Microbe. 2020;1(4):e154-e64.

135. Centre for Genomic Pathogen Surveillance (CGPS). Pathogenwatch collection. Azithromycin resistant gonococcus in global context. https://pa thogen.watch/collection/8mk1i3e55olu-azm-resistant-gonococcus-in-globalcontext. (2020).

136. Wick RR, Holt KE. Benchmarking of long-read assemblers for prokaryote whole genome sequencing. F1000Res. 2019;8:2138.

137. Souvorov A, Agarwala R, Lipman DJ. SKESA: strategic k-mer extension for scrupulous assemblies. Genome Biol. 2018;19(1):153.

138. Spiteri G, Cole M, Unemo M, Hoffmann S, Ison C, van de Laar M. The European gonococcal antimicrobial surveillance programme (Euro-GASP)--a sentinel approach in the European Union (EU)/European Economic Area (EEA). Sex Transm Infect. 2013;89(Suppl 4):iv16-8.

139. Schwarcz SK, Zenilman JM, Schnell D, Knapp JS, Hook EW 3rd, Thompson S, et al. National surveillance of antimicrobial resistance in Neisseria gonorrhoeae. The Gonococcal Isolate Surveillance Project. JAMA. 1990; 264(11):1413-7.

140. Public Health Agency of Canada National Microbiology Laboratory. National surveillance of antimicrobial susceptibilities of Neisseria gonorrhoeae. Annual summary 2017. https://www.canada.ca/en/public-health/services/publica tions/drugs-health-products/national-surveillance-antimicrobialsusceptibilities-neisseria-gonorrhoeae-annual-summary-2017.html. 2017.

141. Lahra MM, Enriquez R, George CRR. Australian Gonococcal Surveillance Programme Annual Report, 2017. Commun Dis Intell. 2018;2019:43.

142. Paine TC, Fenton KA, Herring A, Turner A, Ison C, Martin I, et al. GRASP: a new national sentinel surveillance initiative for monitoring gonococcal antimicrobial resistance in England and Wales. Sex Transm Infect. 2001;77(6):398-401.

143. Weston EJ, Wi T, Papp J. Strengthening global surveillance for antimicrobial drug-resistant Neisseria gonorrhoeae through the enhanced gonococcal antimicrobial surveillance program. Emerg Infect Dis. 2017;23(13):S47-52.

144. Sirivongrangson P, Girdthep N, Sukwicha W, Buasakul P, Tongtoyai J, Weston $\mathrm{E}$, et al. The first year of the global Enhanced Gonococcal Antimicrobial Surveillance Programme (EGASP) in Bangkok, Thailand, 20152016. PLoS One. 2018;13(11):e0206419.

145. Seale AC, Gordon NC, Islam J, Peacock SJ, Scott JAG. AMR surveillance in low and middle-income settings - a roadmap for participation in the global antimicrobial surveillance system (GLASS). Wellcome Open Res. 2017;2:92.

146. Global Health Research Unit (GHRU), Genomic Surveillance of Antimicrobial Resistance. https://ghru.pathogensurveillance.net/ (2020).

147. Cehovin A, Jolley KA, Maiden MCJ, Harrison OB, Tang CM. Association of Neisseria gonorrhoeae plasmids with distinct lineages and the economic status of their country of origin. J Infect Dis. 2020;222(11):1826-36.

148. Gottlieb SL, Ndowa F, Hook EW 3rd, Deal C, Bachmann L, Abu-Raddad L, et al. Gonococcal vaccines: public health value and preferred product characteristics; report of a WHO global stakeholder consultation, January 2019. Vaccine. 2020:38(28):4362-73.

149. Marjuki H, Topaz N, Joseph SJ, Gernert KM, Kersh EN, Antimicrobial-Resistant Neisseria gonorrhoeae Working Group, et al. Genetic similarity of gonococcal homologs to meningococcal outer membrane proteins of serogroup B vaccine. mBio. 2019;10(5):e01668-19.

150. Russell MW, Jerse AE, Gray-Owen SD. Progress toward a gonococcal vaccine: the way forward. Front Immunol. 2019:10:2417.

151. Taylor SN, Marrazzo J, Batteiger BE, Hook EW 3rd, Sena AC, Long J, et al. Single-dose Zoliflodacin (ETX0914) for treatment of urogenital gonorrhea. N Engl J Med. 2018;379(19):1835-45
152. Taylor SN, Morris DH, Avery AK, Workowski KA, Batteiger BE, Tiffany CA, et al. Gepotidacin for the treatment of uncomplicated urogenital gonorrhea: a phase 2, randomized, dose-ranging, single-oral dose evaluation. Clin Infect Dis. 2018;67(4):504-12

153. Institute of Medicine (US) Forum on Microbial Threats. Antibiotic resistance: implications for global health and novel intervention strategies: workshop summary. The National Academies Collection: Reports funded by National Institutes of Health. Washington (DC) 2010.

154. Demczuk W, Martin I, Sawatzky P, Allen V, Lefebvre B, Hoang L, et al. Equations to predict antimicrobial MICs in Neisseria gonorrhoeae using molecular antimicrobial resistance determinants. Antimicrob Agents Chemother. 2020;64(3)

155. Hicks AL, Wheeler N, Sanchez-Buso L, Rakeman JL, Harris SR, Grad YH Evaluation of parameters affecting performance and reliability of machine learning-based antibiotic susceptibility testing from whole genome sequencing data. PLoS Comput Biol. 2019;15(9):e1007349.

156. European Committee on Antimicrobial Susceptibility Testing (EUCAST) clinical breakpoints. https://eucast.org/clinical_breakpoints/. Accessed 2 Dec 2020.

157. Clinical Laboratory and Standards Institute (CLSI) guidelines. https://clsi.org/. Accessed 2nd December 2020

158. Yeats, Corin. Pathogenwatch AMR N. gonorrhoeae library. Gitlab. https://gitla b.com/cgps/pathogenwatch/amr-libraries/-/blob/master/485.toml (2020).

159. Pathogenwatch publication scripts. https://gitlab.com/cgps/pathogenwatch/ publications/-/tree/master/ngonorrhoeae. (2020).

\section{Publisher's Note}

Springer Nature remains neutral with regard to jurisdictional claims in published maps and institutional affiliations.

\section{Ready to submit your research? Choose BMC and benefit from:}

- fast, convenient online submission

- thorough peer review by experienced researchers in your field

- rapid publication on acceptance

- support for research data, including large and complex data types

- gold Open Access which fosters wider collaboration and increased citations

- maximum visibility for your research: over $100 \mathrm{M}$ website views per year

At BMC, research is always in progress.

Learn more biomedcentral.com/submissions 